

\section{AUTEURS PRINCIPAUX}

ASHISH KOTHARI est membre fondateur du groupe environnemental indien Kalpavriksh. II a enseigné à l'Institut indien d'administration publique, a coprésidé le Groupe thématique et orientation stratégique de l'Union internationale pour la conservation de la nature (UICN) sur la gouvernance, les moyens de subsistance et l'équité en relation avec les aires protégées (TILCEPA), et a contribué à la fondation du Consortium mondial pour les APAC.

ROSIE COONEY est présidente du Groupe de spécialistes de l'utilisation durable et des moyens de subsistance (SULi) de la Commission des politiques environnementales, économiques et sociales (CPEES) et de la Commission de survie des espèces (CSE) de I'UICN, et membre du Conseil consultatif scientifique des Nations unies. Elle est consultante en politiques relatives à la biodiversité auprès de gouvernements, d'organisations non gouvernementales et du secteur privé, et donne des conférences à l'Institut d'études environnementales de l'Université de Nouvelle-Galles du Sud.

\section{COLLABORATEURS}

DANNY HUNTER est scientifique principal à Bioversity International et membre de la CMAP-UICN.

KATHY MACKINNON est vice-présidente de la CMAP-UICN et a occupé le poste de spécialiste en chef de la biodiversité à la Banque mondiale, travaillant sur des projets en Afrique, en Amérique centrale et du Sud, en Asie du Sud, en Asie de l'Est, en Asie centrale et en Europe de l'Est.

EDUARD MÜLLER est vice-président pour l'éducation et l'apprentissage de la Commission mondiale des aires protégées de I'Union internationale pour la conservation de la nature, président de l'Université pour la coopération internationale du Costa Rica, Président des réserves de biosphère et biens naturels et mixtes du Patrimoine mondial de l'UNESCO, et coordinateur du Partenariat mondial de la CMAP pour la professionnalisation de la gestion des aires protégées.

FRED NELSON est directeur exécutif de Maliasili Initiatives, une organisation qui promeut la gestion et la conservation durables des ressources naturelles en Afrique. II a contribué à la création du Forum des ressources naturelles de Tanzanie.

KRISHNA PRASAD OLI a travaillé avec le Centre international pour le développement intégré des montagnes (ICIMOD) jusqu'à sa retraite, et avant cela avec plusieurs autres agences, y compris I'UICN, au Népal.

SANJEEVA PANDEY est agent du Service forestier indien et travaille comme Conservateur en chef principal supplémentaire des forêts dans le département forestier de l'Himachal Pradesh, en Inde. II a été directeur du parc national du Grand Himalaya (GHNP).

TAHIR RASHEED travaille actuellement à LEAD Pakistan en tant que directeur général, et a concentré ses efforts sur l'utilisation durable, la recherche sur l'adaptation au changement et les politiques, et la mise en œuvre de projets intégrés de conservation et de développement.

LUBOMIRA VAVROVA est consultante indépendante. Elle a travaillé à la conservation nationale de la nature de la République slovaque, à Banská Bystrica (2002-2006), et au Bureau de programme de l'UICN pour l'Europe du sud-est, à Belgrade (Serbie), en tant que Chargée de projet sur la biodiversité.

\section{REMERCIEMENTS}

Nous remercions Thora Amend, Richard B. Harris, Ernesto Noriega, Tatjana Puschkarsky, Mikko Rautiainen et Vivienne Solis pour leurs contributions à ce chapitre.

\section{CITATION}

Kothari, A., Cooney, R., Hunter, D., MacKinnon, K., Muller, E., Nelson, F., Oli, K. P., Pandey, S., Rasheed, T. et Vavrova, L. Managing resource use and development. In Worboys, G. L., Lockwood, M., Kothari, A., Feary, S. et Pulsford, I. (éd.) Gouvernance et gestion des aires protégées. Canberra : ANU Press, 2020. doi.org/10.22459/GGAP.25

\section{PHOTO DE LA PAGE DE TITRE}

Produits forestiers provenant de forêts conservées par la communauté, Chittagong hill tracts, Bangladesh

Source : Ashish Kothari 


\section{Introduction}

L'utilisation des ressources et les activités de développement de toutes sortes sont monnaie courante dans et autour des aires protégées. Elles entraînent divers impacts sur les valeurs de conservation, sont liées de diverses manières à la vie et aux moyens de subsistance des populations locales et autres secteurs de la société, et sont abordées de différentes manières dans la gouvernance et la gestion des aires protégées. Le présent chapitre donnera un aperçu général des expériences en matière d'utilisation des ressources et de développement à l'intérieur et à proximité des aires protégées.

La première grande partie du chapitre abordera l'utilisation des ressources. Il est généralement admis que l'utilisation durable des écosystèmes et des ressources biologiques peut jouer un rôle important dans la gestion et la conservation des aires protégées. Il existe cependant des questions complexes concernant l'ampleur et le type d'utilisation, ainsi que les types et la fragilité des écosystèmes et des populations sauvages où une telle utilisation se déroule. Les approches classiques de séparation des personnes et des aires protégées, ou autres façons de restreindre l'utilisation des ressources, ont commencé à céder la place à des approches plus inclusives. Ce chapitre aidera à comprendre comment les modèles d'utilisation durable contiennent des éléments tels que des valeurs communes, des rôles, des droits et des responsabilités définis, des mécanismes de résolution des conflits et autres mesures essentielles à une gouvernance équitable et à une gestion efficace des aires protégées. L’approche générale est que la gestion des aires protégées est autant une question de gestion de l'utilisation humaine et de reconnaissance des liens des personnes avec le reste de la nature, qu'une question de caractéristiques intrinsèques des systèmes naturels. Un ensemble de processus reliant l'utilisation des ressources et le développement, les projets intégrés de conservation et de développement (PICD), sera également abordé.

La deuxième grande section abordera la question des projets et processus de développement et d'infrastructures dans et autour des aires protégées. Un aspect de cette situation, celui des projets réalisés par l'aire protégée elle-même, a été abordé au chapitre 24. Le deuxième aspect concerne les activités menées à d'autres fins, telles que la satisfaction des besoins et aspirations des populations dans et autour de l'aire protégée, ou des personnes plus éloignées, y compris les industries extractives et autres, les infrastructures, la production d'électricité, etc. Celles-ci peuvent souvent avoir des impacts négatifs sur les valeurs de conservation des aires protégées et doivent donc être abordées par des réponses juridiques, sociales et de gestion appropriées.

\section{Utilisation des ressources dans et autour des aires protégées}

À travers le monde, les aires protégées ont, pour la plupart, été traditionnellement habitées ou exploitées par l'homme. Les utilisations résidentes, mobiles ou saisonnières des terres, des eaux et des espèces sauvages dans ces aires sont à la fois anciennes et répandues. Il n'existe pas d'évaluation complète, à l'échelle mondiale, mais des extrapolations fondées sur des études indicatives provenant de diverses régions et pays cités ci-dessous donnent à penser qu'une très grande proportion, sinon la majorité, des aires protégées sont susceptibles d'être habitées et/ou utilisées par les populations.

Les chiffres disponibles dans quelques régions ou pays suggèrent que le nombre de personnes utilisant actuellement les ressources dans les aires protégées est d'au moins plusieurs dizaines de millions. Une analyse globale de la situation à la fin des années 1990 a révélé qu'environ $70 \%$ des plus de 30000 sites (alors) inscrits sur la liste des aires protégées des Nations unies autorisaient une utilisation locale des ressources naturelles (Pretty, 2002). En Inde, $69 \%$ des quelque 250 aires protégées étudiées à la fin des années 1980 étaient habitées, et $64 \%$ accordaient des droits communautaires, des baux ou des concessions à l'intérieur de leurs limites. Entre trois et quatre millions de personnes vivent dans des aires protégées, et plusieurs autres millions, dans les zones adjacentes, utilisent les ressources qu'elles abritent (Kothari at al., 1989). Il est peu probable que la situation ait sensiblement changé depuis. Au moins cinq aires protégées ont signalé une population de plus de 100000 personnes à l'intérieur de leurs limites. Près de $90 \%$ des parcs nationaux de 1984, déclarés jusqu'en 1991 en Amérique du Sud, étaient habités ou utilisés (Amend et Amend, 1995). Un nombre important d'aires protégées en Europe abritent également des populations humaines.

Si l'on inclut les "nouveaux " types de gouvernance des aires protégées (voir le chapitre 7) qui ne font pas nécessairement partie du système formel, comme les aires du patrimoine autochtone et communautaire (APAC) et les aires protégées privées (APP), le nombre d'aires habitées ou utilisées augmente encore, ainsi que le nombre de personnes impliquées. Toutefois, il n'existe pas de chiffres complets pour ces types d'aires protégées.

\section{Types d'utilisation des ressources et leur importance pour les populations locales}

L'occupation humaine des aires protégées et l'utilisation des ressources qui s'y trouvent vont d'établissements permanents 
Au Pakistan, les aires protégées fournissent des biens et des services à un grand nombre de personnes (Pakistan Forest Act, 1927 ; Pakistan Wildlife Act, 1974 ; Jan, 1992). Les aires protégées forestières (initialement déclarées pour la préservation des ressources forestières, mais plus récemment également axées sur la conservation de la faune et de la flore sauvages) sont divisées en catégories publiques et privées/communautaires : selon Jan (1992), 66 \% sont des forêts publiques et 34 \% sont privées ou appartiennent aux communautés locales. Certains droits et privilèges sont inclus dans les deux catégories. Les forêts protégées désignées (appartenant à l'État) autorisent certains droits et concessions, notamment le pâturage, la coupe d'herbe et la collecte de bois sec, à moins que ces activités ne soient interdites par le gouvernement.
Les forêts de Guzara (de subsistance) (propriété privée/ communautaire) ont été mises en réserve pour répondre aux besoins authentiques des communautés locales, en particulier dans les régions de Malakand et Hazara au Pakistan. Dans ces forêts, les ayant-droits coutumiers ont droit à $60-80 \%$ des produits de l'exploitation forestière (réalisée par le Département des forêts), ainsi qu'à la coupe d'arbres verts (au moyen de permis) et à la collecte de PFNL. Les ayant-droits coutumiers et autres résidents dans un rayon de 8 kilomètres sont autorisés à ramasser du bois sec et à faire paître leurs animaux. Pour la plupart des aires protégées, des zones tampons ont été créées, principalement pour fournir des produits d'usage ou de valeur (cultures commerciales) à la population locale. en termes d'utilisation durable des ressources à des fins de subsistance. La catégorie IV (Aire de gestion des espèces ou des habitats) s'appuiera parfois sur les modes traditionnels d'utilisation des ressources (par exemple, le pâturage) pour maintenir les valeurs de conservation souhaitées, tandis que le maintien des modes d'interaction entre les humains et les paysages terrestres ou marins par le biais de pratiques traditionnelles est l'un des principaux objectifs de la catégorie V (Paysage terrestre ou marin protégé). Enfin, l'utilisation durable est au centre des aires protégées de catégorie VI (Aires protégées avec utilisation durable des ressources naturelles). Ces sites couvrent aujourd'hui $32 \%$ de la superficie mondiale des aires protégées attribuées à une catégorie de l'UICN (la plus grande), et présentent des niveaux de naturalité ou d'influence humaine similaires à ceux des aires de catégorie II (Parc national) (Bertzky et al., 2012).

\section{Attitudes à l'égard de l'utilisation des ressources dans les aires protégées}

\section{Une histoire d'exclusion : le modèle du Yellowstone}

Dans de nombreuses régions du monde, la création d'aires protégées officielles a suivi le "modèle du Yellowstone ", établi en 1872 avec la déclaration du parc national du Yellowstone aux États-Unis. Ce paradigme était généralement protectionniste et exclusif, avec les croyances sous-jacentes centrales que l'utilisation humaine est nécessairement ou intrinsèquement préjudiciable aux objectifs de conservation, et que l'appareil d'État est l'approche de gouvernance la plus efficace pour atteindre les objectifs de conservation (Neumann, 1988 ; Kothari et al., 1995 ; Adams, 2004). L'approche du Yellowstone, lui-même, ainsi que de nombreuses autres aires protégées, a depuis évolué. Pourtant, l'approche d'exclusion reste endémique dans de nombreuses régions du monde, et les initiatives visant à restituer les droits coutumiers, où ils étaient auparavant confisqués, sont rares.

Les aires protégées gérées par les gouvernements ont souvent été établies sans consultation avec les communautés qui y vivent ou utilisent les ressources qui s'y trouvent. La propriété de la faune et des ressources est généralement légalement dévolue à l'État, ce qui entraîne la détérioration des systèmes fonciers coutumiers, et même, l'expulsion des résidents locaux ou des communautés d'utilisateurs de leurs zones ancestrales : les amérindiens du Yosemite et autres parcs nationaux, les Masaï des réserves désormais emblématiques comme le Serengeti en Tanzanie, les Batwa ( Pygmées ») de Bwindi en Ouganda, les Karen des réserves de Thaillande, plusieurs communautés forestières des réserves de tigres en Inde, les Basarwa ("Bushmen ») du Botswana de la réserve de faune du Kalahari Central (des zones qu'ils occupaient depuis 30000 ans), et bien d'autres encore (Spence, 1999 ; West et al., 2006 ; Dowie, 2009 ; Lasgorceix et Kothari, 2009). Cela n'a fait quajouter à la dépossession et à la marginalisation des communautés aux fins de la colonisation et de l'industrialisation.

Cependant, dans certaines régions, comme en Europe et dans certaines parties du bassin amazonien, l'utilisation des ressources existantes et les populations locales ont été intégrées, dès le début, dans la vision et la gestion des aires protégées, dont des exemples apparaissent plus loin dans ce chapitre.

Les premières lois sur la faune en Afrique du Sud et en Afrique de l'Est visaient en grande partie les colons, dont les actions entraînaient l'épuisement des grands mammiferes (par exemple, par les marchands d'ivoire, les chasseurs sportifs et de trophées), ou le défrichement de zones fauniques pour les établissements agricoles (Anderson et Grove, 1987). Les premières aires protégées en Afrique de l'Est ont maintenu 
les droits fonciers coutumiers des communautés locales et l'utilisation des ressources (Nelson et al., 2007). Au cours de la première moitié du XXe siècle, les politiques en matière d'aires protégées ont évolué vers une position plus exclusive, décourageant la résidence des communautés locales et l'utilisation des ressources. D'importants débats ont eu lieu, avant et après l'indépendance en Afrique del'Est, par exemple, autour de la présence d'éleveurs dans des réserves fauniques clés, telles que le parc national d'Amboseli au Kenya ou le parc national du Serengeti en Tanzanie (Neumann, 1998). Cette évolution vers l'exclusion était souvent davantage motivée par des notions externes, introduites, comme la nécessité d'une " nature sauvage vierge et inviolée ", plutôt que par une analyse empirique des impacts positifs ou négatifs des communautés locales sur la conservation (Homewood et Rodgers, 1991 ; Neumann, 1998), bien que, comme on le verra plus loin, cela ne signifie pas que les communautés aient toujours vécu en harmonie avec leur environnement naturel.

Dans plusieurs pays, où des privilèges d'utilisation des ressources locales ont été maintenus par le gouvernement dans les aires protégées, il s'agit généralement plutôt d'exceptions à la règle, en particulier en ce qui concerne les sites relevant globalement des catégories I, II et IV de l'UICN. Là encore, l'histoire du Serengeti, en Tanzanie, est révélatrice. Lorsque les communautés Massaïs ont été expulsées et que le parc national du Serengeti a été déclaré aire protégée d'exclusion, en 1959, cela était basé sur un compromis avec ces communautés, excluant les hautes terres du Ngorongoro et la partie adjacente des plaines du Serengeti du parc, et les incluant dans la nouvelle aire de conservation polyvalente du Ngorongoro (NCA) (Homewood et Rodgers, 1991). La NCA a été explicitement, et de forme tout à fait unique en Afrique de l'Est, établie comme une aire de conservation gérée par l'État, avec pour mandat d'équilibrer la conservation de la faune et le développement économique local. Les droits coutumiers des Massaï à la résidence et à l'utilisation des ressources, principalement par le biais du pâturage traditionnel du bétail, sont explicitement intégrés dans la charte et les systèmes de gestion de la NCA.

En Afrique du Sud, la communauté Makuleke a obtenu environ 20000 hectares de terres dans le parc national de Kruger en 1998, dans le cadre du processus de revendications territoriales postapartheid et des négociations connexes avec l'Autorité des parcs nationaux (Reid, 2001). Cette revendication n'a toutefois été reconnue que sur la base du fait que l'utilisation des terres de conservation ne serait pas autorisée à changer, et que les Makuleke cèderaient leurs terres à Parcs nationaux d'Afrique du Sud au moyen d'un contrat de parc national. Dans de nombreuses régions d'Afrique, les gestionnaires d'aires protégées restent généralement réticents à intégrer l'utilisation locale ou la cogestion dans les principales aires protégées, en particulier les parcs nationaux (Steenkamp et Uhr, 2000).

Dans le sous-continent indien, il existe une longue histoire de conservation et une grande diversité de modèles de protection, à la fois par les communautés et par les dirigeants. Dans les temps plus modernes, cependant, c'est le modèle du Yellowstone qui a été uniformément adopté pour la déclaration des aires protégées officielles (Saberwal et al., 2001). La biodiversité est souvent concentrée dans des régions où la pauvreté (au sens conventionnel du terme) tend à être omniprésente, et où la portée des programmes de développement gouvernementaux est souvent limitée (Pandey et Wells, 1997). La création d'une aire protégée dans de telles situations entraîne souvent une perturbation de l'utilisation des ressources par la communauté. Entre 100000 et 600000 personnes ont été expulsées physiquement en Inde, et des centaines de milliers d'autres ont été privées de leurs moyens de subsistance (Wani et Kothari, 2007 ; Lasgorceix et Kothari, 2009).

L’Amérique latine présente une histoire plus contrastée. Au Costa Rica, au Chili et en Argentine, des aires protégées ont été créées relativement tôt, principalement sous le concept de " parcs nationaux ", dans lesquels les terres sont principalement la propriété de l'État (bien que dans certains cas, comme au Costa Rica, le gouvernement doive toujours aux propriétaires d'origine le paiement de l'expropriation d'un peu moins de la moitié des terres aujourd'hui inclues dans les aires protégées) (Programa Estado de la Nación, 2006). Au Mexique, seul un faible pourcentage des terres situées dans les aires protégées est la propriété du gouvernement fédéral, avec un pourcentage élevé sous régime communautaire ou privé, ce qui signifie que le système d'aires protégées doit négocier en permanence avec les propriétaires fonciers pour leur protection (BezauryCreel et Carbonell, 2009).

\section{En finir avec l'approche d'exclusion}

La fin du XXe siècle a été témoin d'une réévaluation de certaines de ces approches de conservation de la biodiversité. La planification de la conservation a souvent utilisé des approches "descendantes " et centralisées, qui accordent peu d'attention aux besoins ou aux aspirations des communautés locales (Hunter et Heywood, 2011). Ces stratégies de " commande et contrôle " perpétuaient souvent la pauvreté, les inégalités et les structures de pouvoir, entravant la réalisation des objectifs de conservation de la biodiversité et de bien-être durables. Les communautés locales et autochtones des pays riches en biodiversité sont étroitement liées à leur environnement naturel depuis des millénaires, et possèdent une connaissance intime des habitats et de leurs espèces végétales et animales sauvages, une relation qui a souvent été perturbée par les approches 
conventionnelles de conservation (UN, 2009). Par exemple, la zone maritime territoriale du Costa Rica a exclu les petits pêcheurs et les communautés côtières de l'accès aux terres et aux ressources dont ils dépendent (Fonseca, 2009). De plus en plus, il est reconnu qu'il n'est ni politiquement faisable, ni éthiquement justifiable, de refuser aux communautés locales l'utilisation des ressources naturelles sans leur fournir de moyens de subsistance alternatifs, ou de gérer des aires protégées sans leur autonomisation et leur soutien (McNeely et al., 1990 ; Wells et al., 1992 ; WRI et al., 1992). Dans certains cas, l'utilisation réglementée des ressources peut également fournir un flux de revenus pour la gestion des aires protégées.

De nombreuses bourses d'études sur les biens communs depuis la fin des années 1980, ainsi que des études récentes, ont mis en évidence la capacité des populations locales à gérer de manière durable et efficace les ressources naturelles et les écosystèmes (Berkes, 1989 ; Ostrom, 1990 ; Hayes, 2006 ; Porter-Bolland et al., 2011 ; Nelson et Chomitz, 2011). En aucun cas, ce phénomène n'est universel, et il existe également des preuves de non-durabilité dans plusieurs situations (Terborgh, 2004), y compris les extinctions causées par les peuples anciens, mais il s'agit d'un phénomène suffisamment répandu pour exiger une plus grande attention que celle accordée par les politiques de conservation conventionnelles. Un facteur particulièrement important est la capacité des communautés à établir et à appliquer des règles qui régissent l'utilisation des ressources, ce qui souligne la nécessité de déléguer des droits clairs aux utilisateurs locaux des ressources dans et autour des aires protégées (Chhatre et Agrawal, 2009). Ces résultats scientifiques ont progressivement renforcé la légitimité de formes plus communautaires de gouvernance et de gestion des aires protégées, et ont souligné que, dans de nombreuses situations, les aires protégées à usages multiples ou les terres autochtones peuvent être autant ou plus efficaces que des instruments de conservation plus stricts comme les catégories d'aires protégées I ou II, en particulier sous des pressions comparables (Nelson et Chomitz, 2011). Cela ne veut pas dire que les peuples autochtones et communautés locales sont, dans toutes les situations et dans tous les cas, axés sur la conservation. Beaucoup sont soumis à des influences externes et internes affectant leurs méthodes traditionnelles ou coutumières, et les jeunes générations sont également soumises à de fortes pressions d'attraction et répulsion pour adopter des modes de vie non durables. À toute chose égale, cependant, il semble que l'adoption progressive d'approches participatives fondées sur les droits soit nécessaire à une conservation plus efficace.

En raison de cette dynamique multiforme, l'exclusion stricte de l'utilisation des ressources, si nécessaire et bénéfique dans des situations où cette utilisation est intrinsèquement préjudiciable aux espèces ou aux écosystèmes locaux (de telles situations sont abordées plus loin dans le présent chapitre), peut aussi parfois avoir des effets pervers et préjudiciables. Ceux-ci incluent notamment d'aliéner les communautés locales des efforts de conservation, de supprimer toute incitation à coopérer avec les gestionnaires et la réglementation des aires protégées, de perdre les avantages des connaissances et pratiques de gestion des ressources traditionnelles en matière de conservation et de gestion, d'aggraver l'utilisation illégale, de déplacer l'utilisation des ressources vers d'autres zones, entraînant des impacts plus importants, d'accroître l'utilisation illégale par des " étrangers " en supprimant les droits et la présence des gardiens traditionnels, de bouleverser les réseaux trophiques complexes avec des conséquences imprévues sur les espèces de conservation cibles, et de perdre des options indispensables de financement durable des aires protégées. La suppression des moutons domestiques du projet de parc national de Patagonie est un facteur qui, par inadvertance, pourrait entraîner un déclin de l'espèce que cette initiative vise à protéger : l'huemul (Hippocamelus bisulcus) (Wittmer et al., 2013). L'interdiction du pâturage des buffles dans le parc national emblématique de Keolodeo (Bharatpur), en Inde, aurait entraîné des changements d'habitat préjudiciables à la conservation de la grue de Sibérie, en voie de disparition (Vijayan, 1991), et l'interdiction des incendies de forêt dans une réserve de tigres du sud de l'Inde est connue pour avoir entraîné des impacts écologiques négatifs que les peuples autochtones auraient pu éviter (voir l'étude de cas 8.1 sur le feu et la tribu Soliga).

\section{Changer les paradigmes : une plus grande inclusion et de nouveaux modèles pour la gestion des aires protégées}

Progressivement, la mission des aires protégées par les gouvernements est passée de la conservation de la biodiversité à l'intégration de considérations d'amélioration du bien-être humain. Le résultat est un changement en faveur d'aires protégées permettant une utilisation durable des ressources locales (Naughton-Treves et al., 2005), ou de formes de loisirs, de recherche, d'éducation ou commerciales d'utilisation des ressources profitant aux populations et aux communautés locales. Bien que les aires protégées soient désignées ou destinées principalement à la conservation de la biodiversité, de plus en plus, elles sont également considérées comme des moteurs et fournisseurs d'avantages sociaux et économiques (Brandon et al., 1998).

La participation communautaire est aujourd'hui généralement considérée comme essentielle à la réalisation des objectifs économiques, politiques, sociaux et environnementaux qui sous-tendent la conservation, tandis 
que la conservation exclusive est remise en question pour des raisons sociales, institutionnelles et de durabilité (Saberwal et al., 2001). Les droits et revendications des peuples autochtones et communautés locales sur leurs terres et ressources traditionnellement détenues sont de plus en plus reconnus, ainsi que les effets négatifs que la création d'aires protégées a souvent entraîné sur ces terres (West et al., 2006). Des évaluations mondiales et des analyses comparatives d'études de cas ont souligné que les professionnels et gestionnaires de la conservation considèrent aujourd'hui la participation comme l'un des facteurs de réussite les plus importants pour la gestion (Stoll-Kleemann et Welp, 2008), bien que la participation ne se traduise pas nécessairement toujours en avantages économiques pour les populations locales (Galvin et Haller, 2008).

D'autres considèrent ce changement comme l'abandon d'une approche de "préservation ", visant à isoler et à maintenir la biodiversité dans les aires protégées en excluant les communautés autochtones et locales, en faveur d'une approche plus bioculturelle (les liens inextricables entre la nature et la culture), autorisant les activités humaines dans le cadre du processus, et augmentant ainsi le potentiel de réussite des stratégies de conservation (Hunter et Heywood, 2011) (voir également les chapitres 4 et 23). Maintenir ou permettre diverses formes d'utilisation des ressources dans les aires protégées fera souvent partie de ces approches. Par exemple, la réserve nationale de Niassa, dans le nord du Mozambique, est la plus grande (42 000 kilomètres carrés) du réseau national d'aires protégées pour la faune sauvage, et abrite environ $80 \%$ des éléphants du pays. Elle intègre les aspects d'utilisation locale coutumière, de présence et de coexistence, avec une population résidente d'environ 35000 personnes (Wikipedia, 2014 ; Niassa Carnivore Projet, 2013).

La gestion communautaire des ressources naturelles (GCRN), l'un des modèles inclusifs émergeants les plus courants, représente un passage d'une approche centralisée à une approche plus décentralisée. La GCRN est essentiellement un terme fourre-tout désignant un large éventail de pratiques par lesquelles des institutions collectives locales ou des groupes de personnes, organisés de manière formelle ou informelle, gèrent et utilisent leurs terres, leurs ressources et leurs biens communs. Cela peut ou non impliquer une aire protégée. Une évaluation récente de l'impact des approches de GCRN en Afrique a mis en évidence quelques résultats écologiques, économiques et institutionnels notables (Roe et al., 2009). Toutefois, comme de nombreuses évaluations effectuées au cours des deux dernières décennies l'ont noté, la GCRN dépend, en fin de compte, de la dévolution des pouvoirs et de la propriété sur les terres et les ressources au niveau local, souvent entravée par des obstacles politico-économiques (Gibson, 1999 ; Nelson, 2010 ; de Beer, 2013).

Les Projets de conservation et de développement intégré (PCDI) représentent un sous-ensemble de ces approches plus inclusives, reliant la conservation de la biodiversité, souvent dans ou autour des aires protégées, au développement social et économique local (Wells et al., 1999). Les PCDI ciblent généralement à la fois l'aire protégée (en renforçant sa gestion) et les communautés locales (en offrant des incitations, telles que des possibilités de développement rural, afin de réduire la pression d'activités préjudiciables sur les habitats et les ressources naturelles). Les PCDI ont souvent commencé comme de petites initiatives dirigées par des ONG, mais ont vraiment pris leur envol lorsque les donateurs internationaux ont adopté le concept de lier la conservation à la réduction de la pauvreté. Aujourd'hui, de nombreuses aires protégées utilisent des modèles de PCDI de taille et de portée variables, allant d'efforts axés sur des sites à de grands programmes visant à intégrer la conservation au développement régional (par exemple, voir Cadman et al., 2010). Ils offrent un cocktail presque irrésistible d'avantages perçus et de conservation de la biodiversité, participation accrue des communautés locales, partage plus équitable des avantages et développement économique pour les plus pauvres dans les zones rurales. Certains ont obtenu des succès remarquables et inspirants, mais de nombreux PCDI n'ont pas atteint leurs objectifs de conservation ou de développement (Brandon et al., 1998 ; Hackel, 1999 ; Oates, 1999 ; Wells et al., 1999 ; McShane et Wells, 2004 ; Alers et al., 2007).

Cette expérience mitigée avec les PCDI est illustrée dans le cas de l'Inde. Sur certains sites, les comités d'écodéveloppement ont donné aux villageois des moyens d'information et de participation, créé des groupes de jeunes et de femmes, permis aux villageois d'accéder à des moyens de subsistance et des ressources de développement supplémentaires par le biais de programmes d'administration locale (panchayat), libéré les communautés tribales et autres villageois pauvres des prêteurs sur gage, et renforcé considérablement la coopération entre les communautés et les agents forestiers. Dans la réserve de tigres de Periyar, les récoltants d'écorce de cannelle ont été encouragés à abandonner leurs activités de braconnage et à utiliser leurs connaissances forestières pour guider les touristes. Bien que leurs revenus du tourisme soient inférieurs à ceux des activités illicites, ils ne sont plus en conflit avec le Département des forêts, ni endettés auprès de prêteurs pour couvrir les amendes, et leur position sociale au sein de la communauté a été renforcée (Periyar Tiger Reserve, 2012). Sur de nombreux autres sites, cependant, ces gains ne se sont pas matérialisés. Au niveau national, le programme d'écodéveloppement en cours a été caractérisé par de graves faiblesses conceptuelles, un suivi inadéquat ou inexistant des 


\section{Étude de cas de 25.2 Écodéveloppement dans le parc national du Grand Himalaya, Inde}

Dans les années 1990, des approches de PCDI ont été introduites à divers endroits, en Inde, y compris le parc national du Grand Himalaya (PNGH). Le programme a commencé en 1994 avec un projet d'enseignement et de vulgarisation de la recherche forestière (PEVRF) soutenu par la Banque mondiale, qui comprenait un sous-projet supplémentaire de Conservation de la biodiversité (Pandey et Wells, 1997). À la fin du projet, les gestionnaires du parc ont lancé des programmes axés sur les moyens de subsistance dans la zone tampon, visant à mettre en place des systèmes communautaires alternatifs de gestion des ressources naturelles, et à résoudre les conflits homme-faune grâce à un mode de gestion participatif (Tandon, 2002 ; Pandey, 2008).

Les femmes appartenant aux ménages les plus pauvres, les plus dépendantes des ressources du parc, ont été organisées dans le cadre de programmes de renforcement des capacités en Groupes d'épargne et de crédit pour les femmes (GECF) dans la zone tampon. Près de 1000 femmes de 95 GECF ont bénéficié d'activités génératrices de revenus : lombricompostage, production d'huile d'abricot, produits du chanvre, écotourisme, théâtre de rue et travail salarié. Des mécanismes sont en cours d'élaboration pour que les GECF renforcent les Conseils de village (panchayat) et deviennent durables.

L'approche d'écodéveloppement du PNGH a également été critiquée par des chercheurs et des militants. Aucun processus démocratique n'avait été établi pour déterminer si un parc national, qui, par la loi, exige la suppression de toute utilisation humaine, était la catégorie de conservation appropriée à appliquer ici, aucune approche intégrée de conservation et de moyens de subsistance n'avait été considérée, et les compensation étaient insuffisantes par rapport à la perte de moyens de subsistance due à l'arrêt d'activités telles que la collecte d'herbes (Baviskar, 2003 ; Chhatre et Saberwal, 2006). En outre, alors que les utilisations traditionnelles des populations avaient été stoppées, le parc a connu à un développement hydroélectrique très dommageable. Le processus du PNGH reflète donc les contradictions de l'approche conventionnelle des aires protégées prévalant en Inde (Saberwal et al., 2001).

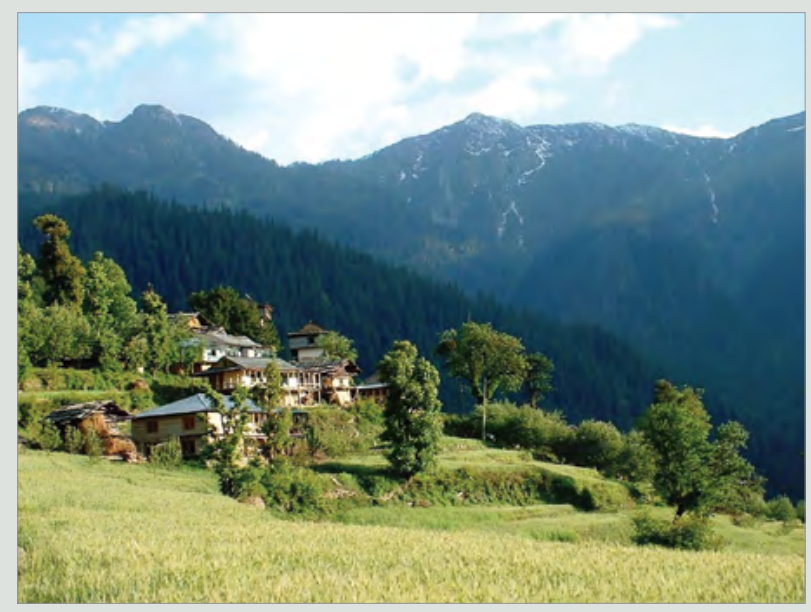

Village dans la zone tampon du parc national du Grand Himalaya, Inde

Source : Sanjeeva Pandey impacts, aucune évaluation indépendante et aucun partage du pouvoir de décision avec les communautés locales (Das, 2007 ; Shahabuddin, 2010 ; voir également l'étude de cas 25.2). Même à Periyar, une étude indépendante suggère que les avantages pour les communautés locales pourraient être moindre qu'officiellement revendiqué (Gubbi et al., 2008).

Les principales faiblesses communes à de nombreuses interventions de PCDI sont les suivantes : objectifs irréalistes et souvent contradictoires, incapacité à identifier correctement la source des menaces et à cibler les interventions en conséquence, suivi insuffisant de sorte qu'il est difficile d'établir un lien efficace entre l'amélioration de la conservation et les activités du projet, et manque de soutien à long terme pour continuer à renforcer les capacités et à maintenir les gains au-delà de la durée de vie du projet (Alers et al., 2007). On peut citer également l'incapacité à identifier et à promouvoir les traditions, les connaissances, les pratiques et les visions du monde autochtones/locales favorisant la conservation, les populations locales étant considérées, la plupart du temps, comme des "pressions " sur l'écosystème et la faune, et l'incapacité à partager de manière significative le pouvoir décisionnel.
La promotion de nouvelles opportunités de subsistance n'est qu'une des façons de profiter aux communautés locales. D'autres stratégies peuvent être plus efficaces pour encourager un soutien à long terme à l'évolution des comportements, y compris des possibilités d'emplois par le biais d'entreprises touristiques (voir le chapitre 23) ou au sein de l'aire protégée, elle-même, en fournissant du travail ou en participant à l'élimination des espèces exotiques envahissantes. Ailleurs, les aires protégées ont tenté de résoudre les problèmes d'équité et de durabilité par le biais de mécanismes de micro-financements ou de financements à long terme, visant à fournir des ressources aux activités de développement, comme par exemple, dans un certain nombre d'aires protégées au Pérou (PROFONANPE, 2012).

Il est essentiel de réaliser un suivi de toutes les activités de PCDI par rapport aux objectifs de réduction des menaces et de conservation de la biodiversité. Dans certains endroits, un lien clair pourra être établi entre une protection renforcée et la conservation, comme par exemple, l'augmentation des stocks de poissons dans les aires marines protégées ou les activités de développement et la protection des habitats. 


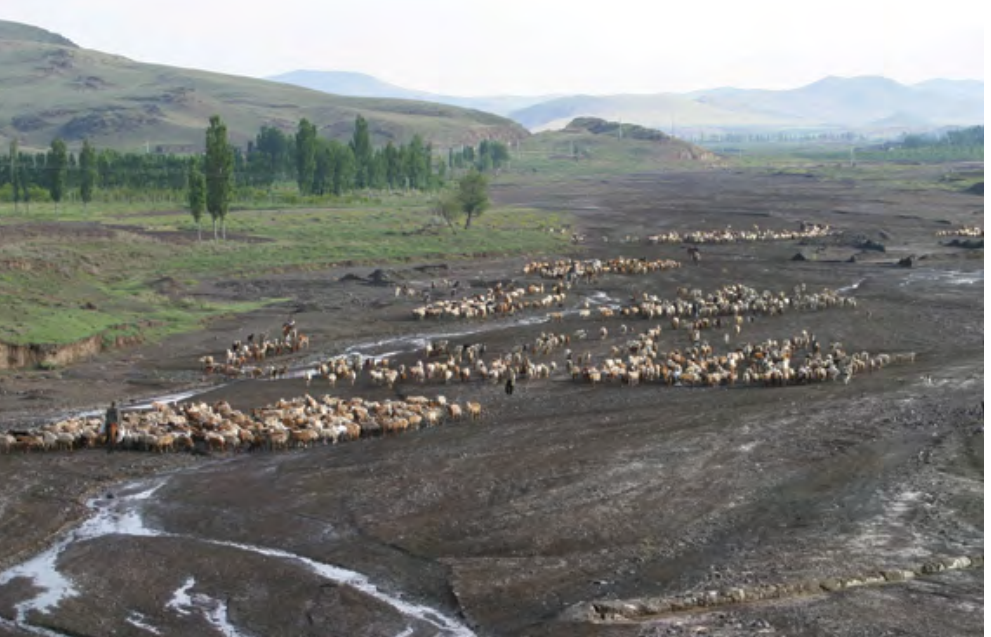

Peuples mobiles en migration à travers leur APAC, Iran

Source : CENESTA

Ailleurs, ces liens pourraient être moins évidents. Lorsque le suivi se concentre davantage sur le nombre de bénéficiaires que sur les résultats de biodiversité et la réduction des menaces, il est de plus en plus difficile de comprendre quand et où les interventions sont efficaces pour la conservation. À moins que les liens entre les activités du projet et les objectifs de conservation ne soient clairs et approuvés par les parties prenantes locales, il est peu probable que l'offre de nouvelles opportunités de subsistance entraine des avantages en matière de conservation. Le suivi participatif par les membres de la communauté peut être un complément utile au suivi par le personnel gouvernemental des aires protégées et les instituts de recherche (Margoulis et Salafsky, 2001 ; Danielsen et al., 2005).

Lutilisation durable des ressources est souvent un objectif clé et un pilier des aires protégées et autres sites de conservation régis par des acteurs non gouvernementaux, y compris les APAC et les APP (voir le chapitre 7). Elle est à la base, par exemple, de milliers de forêts communautaires en Asie du Sud, de centaines d'aires marines gérées localement dans le Pacifique Sud, en Asie du Sud-Est et dans certains pays africains, de vastes territoires de peuples mobiles en Asie centrale et dans la Corne de l'Afrique, ainsi que de nombreuses autres APAC (Borrini-Feyerabend et al., 2010 ; Bassi et Tache, 2011 ; Kothari et al., 2012 ; Naqizadeh et al., 2012 ; voir aussi les chapitres 7, 20 et 21). C'est également une motivation clé pour les APP, tels que celles conservant les grands mammiferes (et la faune associée) dans plusieurs pays africains (voir ailleurs dans ce chapitre).

\section{Reconnaître le rôle de l'utilisation durable dans la conservation}

Il est de plus en plus reconnu qu'une utilisation durable des ressources peut souvent être tout à fait compatible avec les objectifs de conservation, et y contribuer. Dans certains cas, les utilisations humaines traditionnelles ont contribué à façonner les paysages terrestres ou marins d'une manière que les défenseurs de l'environnement considèrent importante, ou peuvent fournir des incitations aux efforts de protection et de conservation, ou générer des revenus indispensables pour financer les aires protégées. Dans certains cas, les récoltes peuvent effectivement augmenter la densité des ressources. Par exemple, dans le désert d'Australie Occidentale, les varans sont plus abondants là où la chasse est plus intense, en raison des techniques de brûlis des parcelles utilisées par les chasseurs autochtones (Bird et al., 2013), bien que les impacts globaux de cette technique sur la biodiversité ne soient pas clairs.

Au Guatemala, la récolte et le commerce durables de petits palmiers (pour une utilisation floristique dans les pays développés) ont permis aux communautés locales de générer des revenus importants, tout en leur offrant des incitations pour maintenir la ressource, préservant ainsi la forêt. Dans la réserve de biosphère Maya, le plus important paysage protégé du Guatemala, les concessions d'utilisation durable sous-tendent un éventail de pratiques de gestion privées et communautaires, conduisant à une conservation plus efficace (Radachowsky et al., 2012). Dans la réserve nationale de faune sauvage d'Ostional, au Costa Rica, des dizaines de milliers de tortues luth arrivent presque simultanément pour nicher chaque année. Les communautés locales sont autorisées à récolter un pourcentage des œufs "précoces ", dont beaucoup auraient été détruits par les arrivées ultérieures. Cette approche a suscité un énorme soutien de la communauté locale en faveur de la conservation, et a pratiquement éliminé le braconnage illégal local des œufs, tandis que la population de tortues continue d'augmenter (Campbell et al., 2007). Au Brésil, la récolte traditionnelle de noix du Brésil dans les forêts amazoniennes, à des fins de rentabilité économique, a entrâné une forte protection de ces forêts par les exploitants contre les bûcherons et les éleveurs (Amazon Conservation Association, 2013).

En Europe centrale, les plaines inondables de la rivière Morava sont des écosystèmes semi-naturels dépendant désormais entièrement de la gestion humaine. Une grande partie de ces plaines inondables (près de 5000 hectares), en Slovaquie, a été inclue dans l'aire protégée du paysage de Záhorie. La forme de gestion la plus appropriée pour maintenir les valeurs de la biodiversité dans ces écosystèmes est la production de foin. Cela empêche les prairies d'être envahies par la végétation et la propagation d'espèces 
envahissantes, et permet de maintenir des biotopes appropriés pour la flore (par exemple, les orchidées) et la faune (par exemple, les papillons) en voie de disparition. Le suivi a confirmé que la biodiversité est significativement plus élevée dans les prairies régulièrement gérées, que dans celles qui ne le sont pas (Rybanič et al., 1999). Le maintien de cette utilisation profite donc à la fois à la conservation de la biodiversité et au développement socioéconomique local. Un autre exemple mettant en évidence la compatibilité de la conservation et de l'utilisation des ressources est le parc naturel de Lonjsko Polje, situé sur les plaines inondables de la Sava, en Croatie. Ce parc est un exemple unique d'un paysage organiquement évolué, conservant des utilisations traditionnelles des terres, avec un système médiéval préservé de pâturages sur des terres communes, typique de toute l'Europe centrale jusqu'à la seconde moitié du XIXe siècle (Gugić, 2009). Ce système d'élevage traditionnel est géré avec des races indigènes de chevaux, de porcs, de bovins et d'oies.

En Irlande, la géologie et le climat locaux uniques du Burren (un paysage karstique dans le nord-ouest du comté de Clare, dont une grande partie est désignée comme Aire spéciale pour la conservation) ont non seulement donné naissance à des paysages uniques, mais ont également contribué au développement d'une forme distincte de transhumance appelée " hivernage », une pratique traditionnelle de pâturage qui a façonné le patrimoine culturel et naturel de la région (Parr et al., 2010). Au cours des 40 dernières années, des facteurs socioéconomiques ont contribué à des changements importants dans l'agriculture, avec des effets néfastes sur la biodiversité. Cette tendance a récemment été inversée par le concept « d'agriculture pour la conservation », qui a ravivé l'intérêt de l'agriculture pour les hivernages, jouant un rôle central dans la restauration du paysage et de sa biodiversité. BurrenLIFE est le premier grand projet « d'agriculture pour la conservation » en Irlande, et marque le premier partenariat de travail entre le Service des parcs nationaux et de la faune sauvage, l'Autorité de développement de l'agriculture et de l'alimentation, et la branche de Burren de l'Association des agriculteurs irlandais (BurrenLIFE, 2014).

En Afrique de l'Est et en Afrique australe, les aires protégées (et leurs populations d'espèces sauvages et conditions d'observation exceptionnelles) servent non seulement à des fins de conservation, mais génèrent également des revenus et des emplois grâce au tourisme. Ces revenus financent, à leur tour, les efforts de conservation, et créent des incitations locales et nationales pour les investissements dans la gestion de la faune (Spenceley, 2008 ; Child, 2004).

Les paysages terrestres et marins de Satoyama et Satoumi, au Japon, connus pour leur utilisation hautement productive des ressources, sont de plus en plus reconnus comme des exemples d'utilisation durable contribuant à la conservation (Bélair et al., 2010 ; UNU-IAS OUIK, 2011).

\section{Principes généraux et approches de l'utilisation des ressources dans les aires protégées}

\section{Droits d'utilisation et gouvernance}

Qui détient les droits d'accès et d'extraction des ressources d'une aire protégée (terrestre ou marine) et qui a le droit de participer à la gestion sont des questions importantes et parfois controversées (voir le chapitre 7). Les droits peuvent découler du régime et des pratiques autochtones, coutumières ou traditionnelles, ou peuvent être développés par le biais de politiques et de lois. Ils peuvent être détenus collectivement ou individuellement, et peuvent être cédés ou transférables de façon permanente par l'achat. Les droits de gestion précisent qui doit participer à la prise de décisions en matière de gestion des aires protégées : ils peuvent être détenus par les gouvernements, par les peuples autochtones et les communautés locales, ou par une combinaison des deux (cogestion). Les droits d'utilisation précisent qui peut avoir accès à une aire protégée ou à une ressource (droits d'accès) et quel degré d'activité (par exemple, le nombre de pêcheurs ou de jours de pêche) ou d'extraction (par exemple, le volume de bois de chauffage ou les tonnes de captures) est autorisé (droits de retrait) (Charles et Wilson, 2009). La reconnaissance appropriée et équitable de ces droits d'utilisation des ressources est de plus en plus considérée comme essentielle pour parvenir à une gestion efficace et durable de l'utilisation des ressources (Charles et Wilson, 2009 ; Charles, 2011).

La Loi de 2006 sur les tribus répertoriées et autres habitants traditionnels des forêts (reconnaissance des droits forestiers) de l'Inde est un exemple de processus en cours de restitution ou de reconnaissance de droits. En vertu de cette loi, les droits individuels et communautaires sur les terres et les ressources forestières qui existaient traditionnellement mais n'ont pas été reconnus depuis l'époque coloniale peuvent être reconnus et consignés. Cela inclut le droit et les pouvoirs de gouverner les forêts. Depuis, 2008, date de l'entrée en vigueur de la loi, ceux-ci ont été reconnus sur plus de 6000 kilomètres carrés de terres forestières (y compris quelques aires protégées par le gouvernement) et, dans plusieurs cas, les communautés élaborent des plans pour les conserver et les utiliser de manière durable, et excluent ce qu'elles considèrent comme des activités destructrices de "développement " et d'exploitation forestière (Vasundhara et Kalpavriksh, 2012 ; Desor, 2013). 


\section{Étude de cas 25.3 Protéger les zones de pêche ancestrales Sangha-Sangha, République Centrafricaine}

Les Sangha-Sangha sont une communauté de pêcheurs située dans la zone tampon du parc national de DzangaNdoki, en République Centrafricaine. En raison de ses valeurs de biodiversité exceptionnelles, la région a été inscrite comme site du Patrimoine mondial tri-national de la Sangha, conjointement avec des parcs limitrophes au Cameroun et au Congo, en 2012. Avec les chasseurs-cueilleurs Baka, les Sangha-Sangha sont les premiers habitants de cette région. Au fil du temps, ils ont développé une relation intime et synergique avec leur territoire, qui a défini leurs valeurs, façonné leur organisation sociale et généré des connaissances environnementales et des systèmes de gestion sophistiqués. Le système interconnecté de canaux et de zones d'inondation que les ancêtres Sangha-Sangha ont créés le long de la rivière Sangha, et qui permet aux poissons de se réfugier et de se reproduire, est un exemple de ceci.

Depuis l'incursion des entreprises forestières dans les années 1980 et 1990, les nouveaux colons ont pratiqué des techniques de pêche non durables, y compris le poison. En réponse à cela, en 2008, les Sangha-Sangha ont créé l'Association pour le développement Sangha-Sangha (ADSS), dans le but de réinstaller la gouvernance locale et les pratiques coutumières. En 2012, l'ADSS a initié un dialogue avec la direction du parc national et autres autorités locales, et a obtenu un décret municipal interdisant l'utilisation de substances toxiques d'origine industrielle et d'équipements non conventionnels pour la pêche, attribuant des droits de pêche exclusifs aux familles ou clans spécifiques reconnus par les autorités traditionnelles, et déclarant que le non-respect de ces dispositions pouvait donner lieu à des poursuites pénales. Les intrusions dans les territoires ancestraux des Sangha-Sangha et les méthodes de pêche non viables ont été érigées en infractions pénales. Depuis, l'ADSS a entrepris des activités visant à promouvoir l'utilisation durable des ressources et à encourager la transmission des connaissances écologiques et des techniques culturelles, en particulier chez les jeunes.

- Ernesto Noriega et Tatjana Puschkarsky

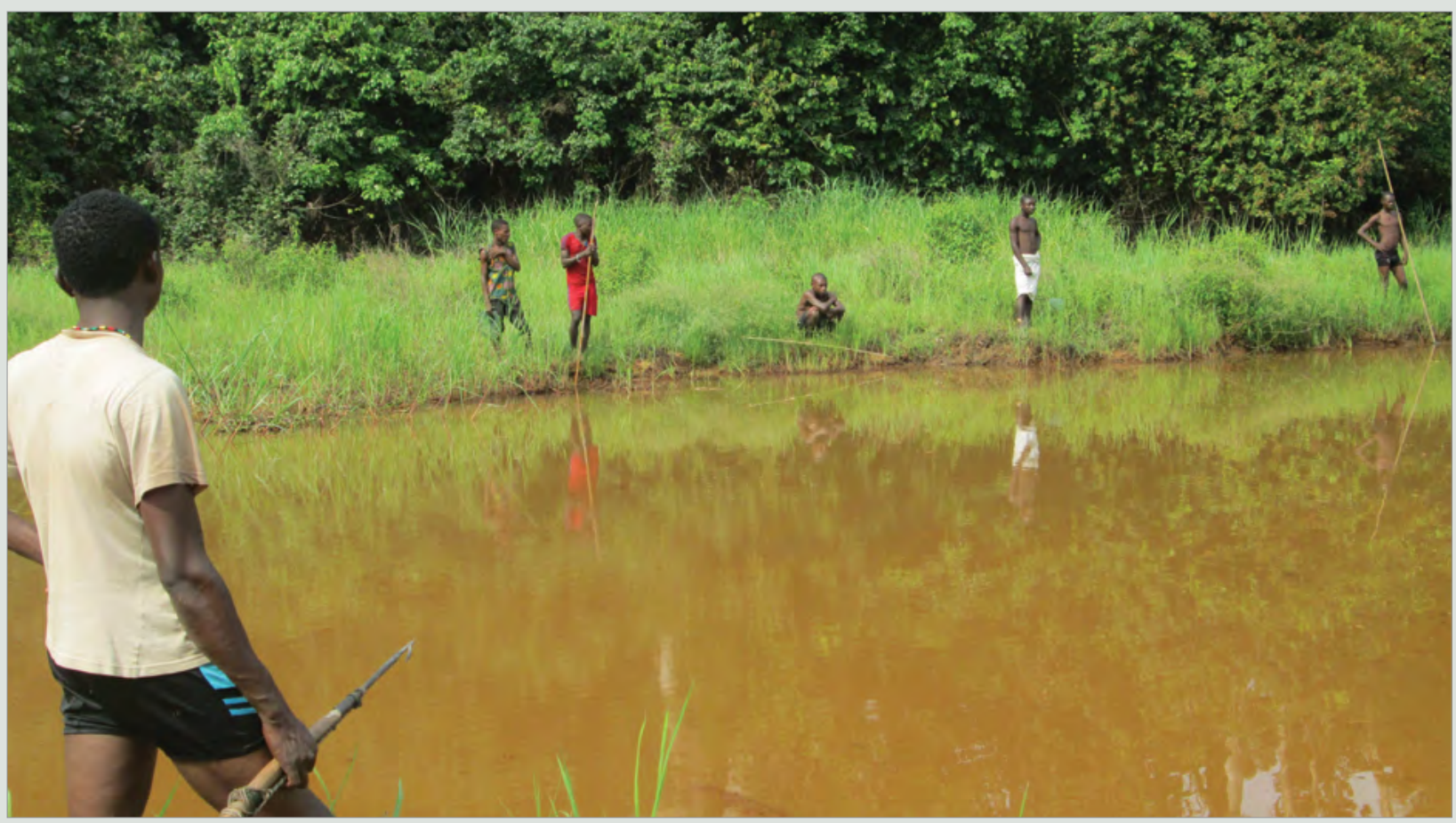

Membres de la communauté Sangha-Sangha dans une zone humide Source : José Martial Betoulet

\section{Connaissances traditionnelles et autochtones}

Une gestion efficace des ressources doit être fondée sur de bonnes informations, susceptibles d'être présentes dans les systèmes de connaissances et pratiques culturelles autochtones et traditionnelles, quand elles sont encore répandues, ou dérivées de méthodes scientifiques occidentales, et idéalement une combinaison de ces éléments (Posey, 1999 ; Failing et al., 2007 ; Fondation Tebtebba,
2008 ; Parrotta et Trosper, 2012). Les connaissances autochtones/traditionnelles et locales peuvent être d'une importance capitale, par exemple pour cartographier les aires d'utilisation des habitats et des ressources, établir des aires d'utilisation des ressources viables et socialement acceptables, élaborer des stratégies axées sur la restauration des paysages, accroître la résilience des écosystèmes et mieux s'adapter aux changements climatiques (voir l'étude de cas 25.3). Cela est particulièrement important dans les aires marines protégées, par exemple, où les utilisateurs 
locaux des ressources ont tendance à posséder une connaissance approfondie de la répartition, de l'abondance et des conditions environnementales des ressources, dans un contexte où ces ressources sont mobiles et où le suivi est difficile (Drew, 2005). À Eastport, sur l'Île de Terre-Neuve, au Canada, la connaissance des pêcheurs communautaires locaux de l'habitat potentiel d'élevage du homard juvénile a servi de base à la prise de décisions sur les zones d'interdiction de pêche au homard, afin d'améliorer la production d'œufs et d'accroître le recrutement (Charles et Wilson, 2009). Une telle approche est typique de nombreuses APAC, et fournit des leçons pour les aires protégées gérées par les gouvernements, privées ou formelles (voir le chapitre 7).

\section{Utilisation durable et équitable}

La gestion de l'utilisation dans un but de durabilité et d'équité est une priorité essentielle pour maintenir les valeurs de la biodiversité. La Déclaration politique de l'UICN sur l'utilisation durable des ressources sauvages (IUCN, 2000) reconnaît quel'utilisation durable est un outil de conservation important, car elle offre aux populations des incitations à la conservation sous forme d'avantages sociaux, culturels et économiques. Ces aspects sont hautement pertinents pour les aires protégées. Ils soulignent également l'importance de la gestion adaptative, les limites biologiques des espèces et des écosystèmes, les structures de gouvernance, le fait que les utilisateurs aient un intérêt formel ou informel dans les ressources qu'ils utilisent et l'élimination des incitations perverses. L'importance d'un régime foncier clair et sûr sur les terres et les ressources, comme base pour motiver les utilisateurs locaux à parvenir à une utilisation durable a également été clairement démontrée par un processus d'analyses régionales et d'études de cas mondiales dirigé par l'UICN (Oglethorpe, 1999).

Dans le cadre de la Convention sur la diversité biologique (CDB), les principes pour parvenir à une utilisation durable (l'un des trois principaux objectifs de la convention) ont été détaillés dans les Principes et lignes directrices d'Addis-Abeba (CDB, 2004). Ces principes et documents connexes fournissent un cadre important pour la gestion de l'utilisation des ressources dans les aires protégées.

Comme pour la durabilité, l'équité socioéconomique est un élément crucial de l'utilisation des ressources. Par exemple, l'intégration des aspects sexospécifiques dans la pensée, la stratégie et la gestion de toute forme d'aires protégées est essentielle, sans quoi l'accès et l'utilisation des ressources par les femmes risquent de subir une marginalisation (FAO, 2012 ; Harper et al., 2013). Dans le secteur de la pêche, l'importance des femmes, en particulier dans les secteurs pré et post-capture, doit être reconnue. Dans les communautés de pêcheurs, les femmes n'ont souvent pas accès au poisson, et peuvent se voir refuser un rôle dans la prise de décisions en raison des normes culturelles existantes, et devoir faire face à des problèmes plus généraux de manque de crédit et de services de transport et de sous-évaluation de leur travail. De même, les inégalités d'accès aux ressources, telles que celles existant entre différents groupes ethniques, classes, castes et autres divisions sociales, peuvent sérieusement entraver l'utilisation durable des ressources et doivent être abordées avec sensibilité.

\section{Gestion de l'utilisation des ressources dans les aires protégées : approches et exemples}

La gestion de l'utilisation des ressources dans les aires protégées doit être très sensible au contexte, et tenir compte de la forme d'utilisation en cause, des caractéristiques de la ressource et du contexte socioéconomique. Dans cette section, certains des principaux types d'utilisation seront discutés, en s'appuyant sur des exemples provenant d'une grande variété de régions.

\section{Récolte de produits végétaux sauvages (utilisation locale et commerce)}

Les forêts, zones humides, prairies et milieux marins protégés sont source d'une vaste gamme de produits forestiers non ligneux (PFNL), définis comme toute matière biologique, autre que le bois rond industriel et les produits qui en résultent, récoltés à l'intérieur et en lisières des forêts naturelles, modifiées ou perturbées (Chamberlain et al., 2004). Bien que le terme puisse inclure à la fois des produits végétaux et animaux, cette section se concentre principalement sur les produits végétaux, les produits animaux étant abordés dans la section suivante. Les PFNL ont une grande valeur économique et de subsistance. Par exemple, Schippmann et al. (2006) estiment que jusqu’à 70000 espèces de plantes supérieures (environ $20 \%$ de la flore mondiale estimée) sont utilisées comme médicaments dans le monde entier, dont environ 3000 font l'objet d'un commerce international. En outre, on estime que pour $80 \%$ de la population mondiale, les plantes constituent la principale forme de médicament disponible (Kamboj, 2000 ; Parrotta et Trosper, 2012). Rien qu'en Inde, environ 275 millions de personnes dépendent des PFNL, et utilisent plus de 10000 espèces de plantes et d'animaux pour leur alimentation, combustibles, fourrage, médecine, logement, outils et utilisations culturelles (TPCG et Kalpavriksh, 2005). La valeur mondiale des PFNL en 2005 s'élevait à 16839 milliards de dollars américains (FAO, 2010). Cela inclut une utilisation extensive et généralisée dans et autour des aires protégées. 
Les récoltants de PFNL sont de plus en plus impliqués dans des entreprises commerciales motivées par la demande du marché national et mondial, les structures de gestion traditionnelles s'effondrant et menaçant, en conséquence, la durabilité de la base de ressources. Par exemple, l'industrie de la santé alternative en Europe, en Amérique du Nord, en Australie et en Nouvelle-Zélande utilise une grande variété de médicaments à base de plantes, et a adopté de nombreuses pratiques de médecines traditionnelles ayurvédiques, bouddhistes et chinoises, de sorte que le secteur est devenue une industrie de plusieurs milliards de dollars, et à croissance rapide. La récolte de plantes médicinales sauvages est ainsi devenue une entreprise commerciale organisée dans de nombreux endroits, où les acteurs du secteur emploient des populations locales pour effectuer la récolte (Battharai et al., 2003). Ceci constitue également une préoccupation pour d'autres produits sauvages, tels que l'ivoire, avec une augmentation récente significative du braconnage pour répondre à la demande de consommateurs nouvellement enrichis en Asie (CITES, 2013).

Les hotspots de biodiversité végétale se trouvent généralement dans les pays à faible indice de développement humain (IDH), sous les tropiques, où la pression pour augmenter le développement économique et humain peut être élevée. Dans de nombreuses aires protégées, les gestionnaires sont chargés de déterminer si la récolte, le pâturage ou autres utilisations à long terme des plantes auront des implications bénéfiques, préjudiciables ou neutres pour la réalisation des objectifs de gestion. Dans d'autres, il s'agit d'une responsabilité assumée par les communautés d'utilisateurs ou les organisations de la société civile. Dans certains cas, il existe des systèmes bien établis et scientifiquement rigoureux de mesure, de suivi, d'évaluation et d'établissement de rapports sur ces activités (voir le chapitre 28). Il est également nécessaire de déterminer quel niveau de l'activité est durable et établir des limites, ce qui est plus efficace avec la participation des communautés d'utilisateurs. Par exemple, la récolte d'herbe dans une zone humide peut être bénéfique pour l'habitat des oiseaux, mais l'augmentation ou la diminution de la récolte ou la modification de la façon dont celle-ci est effectuée peuvent nuire à l'activité. Dans la mesure du possible, un suivi continu des conditions environnementales et sociales visant à évaluer l'impact de ces activités est souhaitable. Un certain nombre d'aires protégées et d'APAC cogérées à travers le monde utilisent des méthodes de suivi allant d'indicateurs traditionnels et de points de référence souvent basés sur des siècles d'observation, à des indicateurs modernes, souvent mieux quantifiés (étude de cas 25.4).

Des mesures visant à éviter la surexploitation sont intégrées dans de nouveaux outils d'évaluation de la durabilité de la collecte des plantes médicinales et autres plantes récoltées à l'état sauvage, comme la norme FairWild, et autres méthodes visant à évaluer à la fois les aspects écologiques et sociaux (FairWild, 2009 ; Kathe et al., 2010 ; Kathe, 2011 ; Unnikrishnan et Suneetha, 2012). Les plans de gestion des espèces constituent également un mécanisme de suivi et de prévention de la surexploitation (études de cas 25.4 et 25.5). Les efforts de politique internationale autour de l'utilisation durable des ressources végétales incluent, entre autres, les Lignes directrices sur la conservation des plantes médicinales (en cours de révision) de l'Organisation mondiale de la santé (OMS), et la Stratégie mondiale pour la conservation des plantes dans le cadre de la CDB (Unnikrishnan et Suneetha, 2012). Il peut être nécessaire de négocier avec les communautés pour mettre fin aux pratiques nuisant à l'aire protégée dans le cas des aires protégées gérées par les gouvernements. Dans le cas des APAC, ces négociations sont généralement menées à l'interne, par les membres de la communauté, et peuvent impliquer de faire face à des pressions internes et externes (étude de cas 25.3). Les nouvelles approches de sauvegarde et de conservation des plantes médicinales incluent la création d'Aires de conservation des plantes médicinales (ACPM) et de Parcs de conservation des plantes médicinales (PCPM) en Inde (Unnikrishnan et Suneetha, 2012). En 2012, 112 ACPM avaient été établies dans 13 États indiens. D'autres stratégies incluent la limitation de l'extraction des ressources à certaines zones, l'autorisation à des personnes spécifiques de récolter la ressource, l'établissement de quotas basés sur un rendement durable et la plantation d'espèces convoitées en dehors de l'aire protégée.

\section{Chasse et pêche}

Un certain nombre de formes de chasse et de pêche ont lieu dans certaines aires protégées, légalement et illégalement, à des fins de subsistance et à des fins commerciales. Le gibier sauvage a longtemps été important pour les communautés rurales, et de nombreuses aires protégées autorisent une chasse et une pêche de subsistance limitées. La viande de brousse est un terme couramment utilisé pour décrire la viande issue de la chasse aux animaux sauvages, principalement dans les environnements forestiers, dans les pays où le bétail domestique n'est pas commun. C'est aujourd'hui une activité commerciale et de subsistance importante en Afrique et, dans une moindre mesure, en Amérique du Sud et en Asie. Dans certaines régions, elle assure la majorité des besoins humains en protéines et en graisses, comme dans le bassin du Congo. Impliquant un coût matériel peu élevé, les jeunes hommes des communautés pauvres peuvent facilement y prendre part, et le commerce décentralisé signifie qu'une grande partie de la valeur des biens revient au producteur primaire (le chasseur) (Nasi et al., 2008, 2011 ; van Vliet et al., 2012 ; Schulte-Herbrüggen et al., 2013). 


\section{Étude de cas 25.4 Aborder le problème de la surexploitation par la négociation et l'action communautaire en Ouganda et en Inde}

Le parc national de Kibale, en Ouganda (catégorie UICN non définie) illustre le succès d'une approche de négociation. Le parc est entouré de 27 paroisses dans lesquelles vivent environ 120000 personnes. Les communautés limitrophes extraient plus de 20 produits du parc, pour répondre à certains de leurs besoins de subsistance, commerciaux, culturels et médicinaux. Bien que l'interdiction ait été la première stratégie de gestion mise en place, il a été constaté que l'application de la loi devenait très longue et coûteuse pour les gestionnaires du parc. II a été constaté que la plupart des activités illégales provenaient des communautés situées en lisière du parc. Avec l'aide du Projet de conservation et de développement de Kibale Semuliki, des accords de gestion des ressources ont été négociés avec les communautés locales adjacentes, fixant des limites convenues sur les personnes autorisées à prélever dans le parc, ainsi que les produits pouvant être prélevés. II a fallu deux ans pour identifier, négocier et signer les premiers contrats, puis six mois, en moyenne, pour les accords suivants. Le succès des accords de collaboration fut plus important lorsque des aides étaient accordées pour l'élaboration de solutions alternatives à la récolte des ressources du parc. Les relations entre le parc et les communautés se sont améliorées, une baisse importante des activités illégales a été constatée, et les membres des communautés ont commencé à signaler les activités illégales (Chhetri et al., 2003).

Le village de Mendha-Lekha en Inde, et sa forêt conservée par la communauté de près de 2000 hectares, a entrepris un certain nombre d'initiatives assertives pour recouvrer les droits forestiers de la communauté et empêcher une usine de papier d'épuiser les habitats locaux de bambou. Après l'obtention d'un titre légal en vertu de la Loi sur les droits forestiers de 2006, le village a renforcé et actualisé ses règles et règlements coutumiers, afin d'inclure l'exploitation durable de bambou (auparavant sous contrôle du Département des Forêts). Aujourd'hui, le village en tire des revenus substantiels et l'argent est déposé sur le compte du village, et est utilisé pour générer des moyens de subsistance pour celui-ci, grâce à des activités liées à la mise en valeur des forêts, à la désignation des habitats fauniques et autres activités. Le village est aujourd'hui en mesure de fournir des salaires équitables et des prêts en temps opportun, non seulement aux résidents, mais aussi aux autres villageois souhaitant travailler dans le village (à condition qu'ils respectent les règles locales) (Pathak Broome et Dash, 2012).

\section{Étude de cas 25.5 Plan de gestion des espèces de Cinnamomum capparu- coronde}

Cinnamomum capparu-coronde est une espèce médicinale endémique hautement menacée au Sri Lanka. La réserve de biosphère de Kanneliya-Dediyagala-Nakiyadeniya (KDN), dans le sud du Sri Lanka, accueille des populations importantes, connues localement sous le nom de "Kapuru Kurundu ». II existe 78 villages autour de la réserve, et $50 \%$ des ménages vivent en dessous du seuil de pauvreté et dépendent de la forêt pour le bois et les PFNL. Cinnamomum capparu-coronde est utilisée localement pour guérir la bronchite, les rhumatismes, les morsures de serpent, les fractures et les maux de dents, entre de nombreux autres maux. L'eugénol est un ingrédient chimique important extrait de la plante.

Dans le but d'empêcher la surexploitation de cette importante espèce endémique, la réserve forestière de KDN a été choisie pour élaborer et mettre en œuvre un plan de gestion des espèces. L'objectif est de maintenir la population de Kapuru Kurundu en réalisant un suivi des changements de densité de 2009 à 2019, dans deux macro-parcelles situées à l'intérieur la réserve de KDN. Le plan est mis en œuvre par le Département des forêts, avec l'aide des communautés locales et des chercheurs de l'Université de Ruhuna et autres organismes. Les besoins économiques et culturels plus généraux des communautés vivant à la périphérie de la réserve ont également été pris en compte par le Département des forêts dans le Plan de gestion de la réserve, auquel le plan de gestion des espèces est lié.

Sources : Sathurusinghe et al. (2010) ; Hunter et Heywood (2011)

des populations. Dans le parc national de Cuc Phuong, au Vietnam, la chasse illégale a réduit les populations de grands mammiferes, et les conflits avec les populations humaines locales entravent une gestion efficace (Compton et Le, 1998 ; McNeely, 1998). De nombreuses aires protégées aquatiques sont confrontées à des problèmes de surpêche causés par des incursions de communautés voisines, ou par la présence illégale d'opérations à plus grande échelle. Sur la Grande Barrière de corail, en Australie, le chalutage de crevettes à grande échelle, à la fois autorisé et illégal, a

Les forces du marché peuvent créer une valeur pour la faur sauvage et fournir des incitations à la conservation privée ou communautaire, mais aussi conduire à la surexploitation 


\section{Étude de cas 25.6 Gestion de la chasse dans les aires protégées appartenant à l'État dans le nord de la Finlande}

Dans le nord de la Finlande, y compris les régions de Laponie, de Kainuu et certaines parties de l'Ostrobotnie du Nord, la chasse est autorisée pour les résidents locaux dans la plupart des parcs nationaux et autres réserves naturelles protégées, si elle ne menace pas les objectifs de conservation ou de loisirs. Dans le nord de la Finlande, environ 50000 personnes bénéficient de tels droits. La chasse est également autorisée pour les non-résidents, avec un permis, dans la plupart des autres réserves naturelles protégées. La chasse est interdite dans les réserves naturelles intégrales.

Les règlements de chasse sont décrits dans le décret ou la loi de chaque réserve naturelle. Des restrictions temporelles ou territoriales, et spécifiques à une espèce, peuvent s'appliquer. Les plans de gestion sont préparés avec la participation des parties prenantes locales. Des saisons de chasse nationales ou régionales sont établies pour toutes les espèces de gibier, en dehors des périodes de reproduction ou de vulnérabilité. Si une population est menacée, la saison de chasse est limitée par un décret du ministère de l'Agriculture et des Forêts.

Les principales parties prenantes disposant de droits d'utilisation traditionnels sont les éleveurs de rennes, représentés par les associations d'éleveurs et leur fédération nationale (leur territoire, dans l'extrême nord de la Finlande, couvre un tiers du pays), et les Sami, dont le territoire traditionnel se situe dans ces régions. Plus de $90 \%$ de cette région est détenue par l'État et administrée par le Metsähallitus, l'Agence nationale finlandaise des aires protégées. Les Sami possèdent une longue tradition d'élevage de rennes et de piégeage du lagopède des saules (Lagopus lagopus). Les associations de gestion du gibier représentent les intérêts des chasseurs résidents.

Les résultats des recensements de la faune montrent des fluctuations naturelles et une stabilité à long terme des populations de gibier dans le nord de la Finlande. Sur cette base, le système de gestion de la chasse peut être considéré comme écologiquement durable. Les plus grands défis concernent la durabilité sociale, affectant le travail bénévole dont dépendent les recensements triangulaires de la faune et les mesures de contrôle des prédateurs envahissants, comme le chien viverrin (Nyctereutes procyonoides) et le vison d'Amérique (Neovison vison).

- Mikko Rautiainen réduit de moitié les populations de certaines espèces. Pour chaque tonne de crevettes capturées, 6 à 10 tonnes d'autres espèces marines ont été tuées. L'organisme de recherche du gouvernement australien, la Commonwealth Scientific and Industrial Research Organisation (CSIRO), a identifié 50 opérateurs illégaux dans une zone de 362400 kilomètres carrés (Australian Committee for IUCN, 1999 ; Zinn et Vidal, 1999).

Lorsque de telles activités ont été interdites ou réduites, une indemnisation a parfois été offerte pour le manque à gagner (en particulier si l'utilisation était légale). C'est le cas de la récolte de plantes médicinales dans l'Himalaya indien (voir l'étude de cas 25.2). C'est aussi souvent le cas dans les APAC, où une décision collective d'arrêter une activité d'utilisation des ressources, ou de changer l'utilisation des terres, est compensée par de nouveaux moyens de subsistance, tels que le tourisme communautaire ou la mise à disposition de terres dans une autre zone. Dans la zone humide protégée par la communauté de Mangalajodi, en Inde, une décision communautaire inspirée par une organisation de la société civile pour arrêter la chasse aux oiseaux d'eau a été suivie par une entreprise d'écotourisme, qui a employé certains des anciens chasseurs (Kothari, 2010). Dans de tels cas, cependant, les mesures compensatoires peuvent ne pas correspondre à l'ampleur de la perte.

Lorsqu'elle est bien gérée, la chasse peut être durable et contribuer à la gestion et à la conservation des aires protégées (étude de cas 25.6), et il est de plus en plus reconnu que pour faire face au problème de la viande de brousse, comme en Afrique, il est nécessaire d'établir une utilisation durable légalement réglementée des ressources en viande sauvage
(Nasi et al., 2008). Dans certains cas, cela a été possible grâce à des accords avec les communautés locales et/ou en confiant la gestion de la faune à ces communautés. Dans bon nombre d'APAC relativement nouvelles, un mélange de restrictions traditionnelles et nouvelles peut être adopté (voir le chapitre 7). Dans l'État du Nagaland en Inde, par exemple, plusieurs dizaines de villages ont déclaré des interdictions saisonnières de chasse et/ou désigné des zones forestières où la chasse est totalement interdite (Kothari et Pathak, 2005). Par exemple, la réserve privée de chasse de Campbell, exploitée commercialement en Afrique du Sud, offre des expériences de chasse inspirées de celles des Bushmen du Kalahari, dans des limites durables (Campbell Private Game Reserve, 2004, cité dans Lockwood et al., 2006).

La chasse touristique ou " au trophée » aux tarifs substantiels, est entreprise dans de nombreuses formes d'aires protégées, dans toute l'Afrique subsaharienne, l'Afrique australe et la Tanzanie en particulier. La majeure partie de ces activités de chasse au trophée se déroulent sur des terres privées et sur certaines terres communales, voire, pour certaines, dans des aires protégées gérées par l'État. En Tanzanie, environ la moitié de toutes les concessions de chasse sont situées dans des aires protégées d'État, appelées réserves de gibier, où aucune personne ne réside, ainsi que dans des aires contrôlées pour le gibier (GCA), où la présence et l'utilisation humaines ont récemment été interdites en vertu de la Loi de 2009 sur la conservation de la faune. Cette disposition de la Loi de 2009 est extrêmement problématique, car les GCA n'étaient pas, avant 2009, des aires protégées exclusives, et abritent, à l'échelle nationale, entre 500000 et un million de personnes. En 2013, un conflit majeur a éclaté 
au sujet de la GCA de Loliondo, suite à une proposition du gouvernement selon laquelle 1500 kilomètres carrés d'anciennes terres communautaires deviendraient une GCA exclusive ou un " corridor faunique " (Ngoitiko et Nelson, 2013). Ces luttes autour de la conservation de la faune sauvage, des utilisations commerciales telles que la chasse, et des droits locaux de propriété de la terre et des ressources sont débattues, en Tanzanie, depuis 50 ans. La chasse dans les réserves de gibier est basée sur une division de ces aires protégées en " blocs » ou zones de concession, et un quota annuel pour chaque bloc est accordé par le ministère des Ressources naturelles et du Tourisme. La chasse fournit une justification économique importante pour conserver ces terres comme habitat faunique, bien que des faiblesses considérables en matière de gouvernance et de réglementation de la chasse, y compris la corruption, soient évidentes (Leader-Williams et al., 2009 ; Nelson et al., 2013).

En Namibie, selon le modèle de conservation communautaire, les communautés génèrent des revenus grâce au tourisme photographique et de chasse, à la vente de gibier vivant et à l'" élevage de gibier " pour la viande et les peaux. Cette approche a considérablement accru la valeur sociale et économique de la faune pour les populations, changeant les attitudes et conduisant à des changements à grande échelle dans l'utilisation des terres, convertissant des terres pastorales dégradées en zones de conservation de la faune, et entraînant un rebond des populations d'espèces telles que l'éléphant et les rhinocéros noir et blanc (Naidoo et al., 2011 ; Nelson et al., 2013).

La chasse au trophée du gros gibier au Pakistan est apparue comme une approche de conservation contribuant à améliorer les moyens de subsistance locaux (Frisina, 2000 ; Frisina et Tareen, 2009). Markhors, urials et bouquetins sont quelques-unes des grandes espèces sauvages présentes dans différentes parties du Pakistan, et disposant d'un marché international pour la chasse au trophée. Cependant, les populations de ces espèces, entre autres, sont en déclin depuis l'époque coloniale, en raison de la chasse à grande échelle et de la perte d'habitats. Lidée d'une chasse au trophée organisée et légale comme moyen d'inverser ce déclin a été développée pour la première fois par le Programme de soutien rural Agha Khan, le WWF-Pakistan et la Society for Torghar Conservation Protection. La chasse au markhor a commencé en 1997, lorsque la Convention sur le commerce international des espèces de faune et de flore sauvages menacées d'extinction (CITES) a approuvé un quota. Réglementée par les ministères nationaux et provinciaux de la faune en vertu de la loi, $80 \%$ des recettes de la chasse au trophée sont reversées aux communautés locales. Les anciens de la communauté, par le biais d'une jirga traditionnelle (réunion des anciens), peuvent interdire

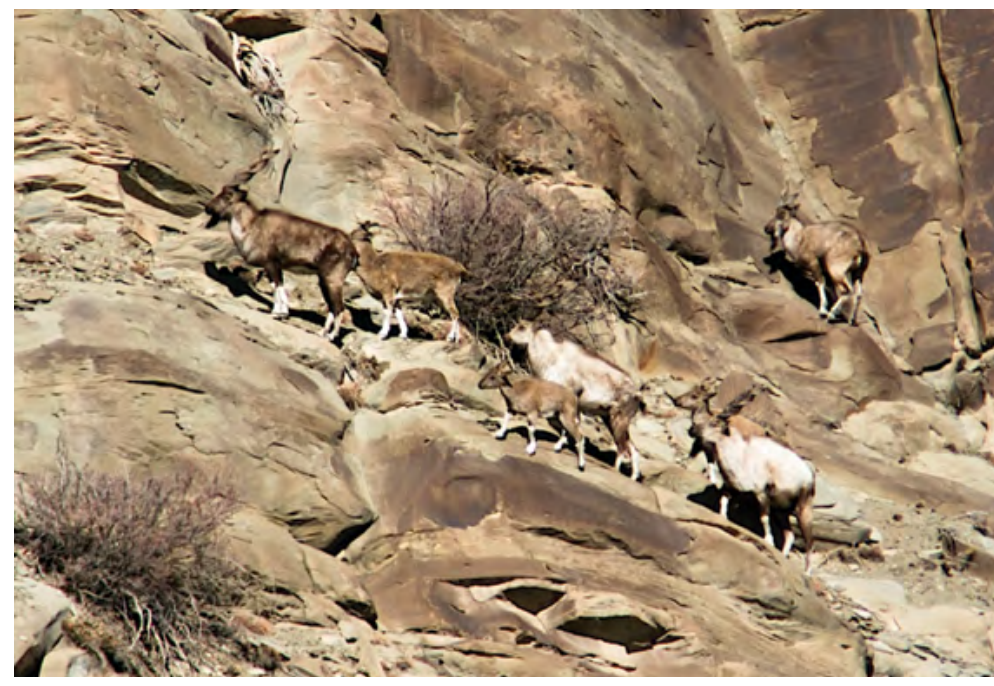

Markhor (Capra falconeri) dans l'aire de conservation communautaire de Torgarh, au Baloutchistan, Pakistan

Source : Tahir Rasheed

toute activité commerciale ou chasse illégale dans leur région, et établir des règles pour une répartition équitable des recettes. Avec des revenus substantiels pour des avantages collectifs, les attitudes locales sont progressivement devenues plus positives à l'égard de la conservation. Il y avait environ 200 urials (Ovis arientalis) et moins de 100 markhors (Capra falconeri) dans la région de Torghar, au Baloutchistan, lorsque la jirga a décidé d'essayer cette approche. En 2005, la population de markhors avait augmenté à 2540 individus, et celle des urials à 3145 (Arshad et Khalid, 2008).

Aux États-Unis, la gestion de la chasse, indépendamment de la catégorisation en tant que sport, loisir, trophée ou subsistance, est généralement du domaine de chaque État, tous disposant d'organismes financés par des fonds publics chargés de cette responsabilité (Bolen et Robinson, 2003 ; United States Fish \& Wildlife Service, 2014). Les tribus amérindiennes reconnues au niveau fédéral gèrent la chasse séparément des gouvernements des États, et la plupart ont leurs propres agences de gestion.

\section{Agriculture}

L'agriculture de subsistance et l'agriculture de marché sont largement pratiquées dans certains types d'aires protégées, en particulier (mais pas seulement) la catégorie V de l'UICN (Amend et al., 2008). Les systèmes de culture et d'élevage sont souvent complétés par des ressources provenant d'écosystèmes naturels. Les forêts et zones humides environnantes sont utilisées à diverses fins, y compris la collecte de litière de feuilles, de produits antiparasitaires, de médicaments, de nourriture, de fourrage et de combustibles.

La culture itinérante, ou swidden, est largement pratiquée comme une forme d'agriculture de subsistance à travers 


\section{Étude de cas 25.8 Aire de gestion marine de la Soufrière, Sainte-Lucie}

L'aire de gestion marine de la Soufrière, à Sainte-Lucie, est une zone à usages multiples comprenant des réserves marines sans pêche, des zones de pêche prioritaires et autres zones d'utilisation. Avant sa création, en 1994, de nombreux conflits existaient entre les pêcheurs traditionnels locaux, les touristes, les visiteurs d'un jour et les propriétaires de yachts naviguant dans les Antilles. Des plongeurs en visite faisaient des trous dans les casiers de pêche pour libérer les poissons de récif, des plaisanciers ancraient leurs embarcations dans des baies sablonneuses, interférant avec la pêche locale de poissons pélagiques côtiers, et l'accès à la plage et à la mer était limité par des installations touristiques. Les populations de poissons de récif étaient menacées par la pêche au harpon et la pêche au casier illégales, et les ancres endommageaient le récif (Salm et al., 2000).

En 1992, le ministère de la Pêche et l'Institut des ressources naturelles des Caraïbes ont entamé des négociations, un processus de règlement des conflits et une planification participative. Une cartographie de toutes les utilisations a été entreprise et un accord préliminaire a été conclu pour le zonage de 11 kilomètres de littoral. Cependant, la mise en œuvre de ce programme n'a été couronnée de succès que pendant deux à trois ans, après quoi elle a été interrompue en raison de violations commises par certaines parties, l'accord n'ayant aucune base légale. Après une évaluation institutionnelle complète, un nouveau régime de gestion a été élaboré sur la base d'une mission clairement convenue, d'une structure de gestion transparente et d'une base juridique solide (Salm et al., 2000 ; Geoghegan et Renard, 2002).

En 2005, l'aire de gestion marine de la Soufrière a célébré son dixième anniversaire. Une étude antérieure a montré que la biomasse de poissons commerciaux dans la réserve marine avait quadruplé et que la zone de pêche avait triplé. Le site était devenu financièrement autonome, grâce aux revenus générés par la plongée et l'amarrage de yachts. La capacité institutionnelle a augmenté dans tous les groupes de parties prenantes, et le tourisme a été bénéfique pour la communauté locale (Gell et Roberts, 2002). Des défis continuent de se poser, mais toutes les parties prenantes se sont engagés à y faire face.

\section{Pâturage et pastoralisme}

Des études ont montré que certains niveaux de pâturage sont durables, et qu'ils peuvent également être essentiels au maintien de certaines prairies très diversifiées, la suppression des personnes et du bétail entraînant une diminution de la biodiversité dans l'aire protégée (Infield, 2003 ; Parr et al., 2010 ; Nelson, 2012). En Afrique et en Asie occidentale, les éleveurs nomades ont fait paitre durablement leur bétail dans certaines régions pendant des siècles. Lorsque les cultures traditionnelles étaient basées sur l'élevage du bétail, les systèmes qu'elles ont établis sur de longues périodes ont souvent façonné le paysage, leur utilisation devenant partie intégrante du maintien des processus écologiques et de la biodiversité (Farvar, 2003 ; Borrini Feyerabend et al., 2004; voir également les références dans la section "Agriculture ", ci-dessus). En Europe, une grande partie de la biodiversité dans les aires protégées s'est développée conjointement avec les pratiques traditionnelles d'élevage (étude de cas 25.7).

Même lorsque le pâturage ne fait pas partie d'un paysage bioculturel établi de longue date, il peut parfois être utile pour atteindre les objectifs de gestion des aires protégées. Par exemple, au Costa Rica, le pâturage a été utilisé dans la restauration de l'écosystème forestier tropical sec du parc national de Guanacaste, pour la dispersion des graines, le contrôle des graminées exotiques et l'obtention d'un soutien local (Evans, 1999). Dans le refuge de Palo Verde, également au Costa Rica, le pâturage du bétail a soutenu la conservation d'une zone humide particulière (Vaughan et al., 1996).

Toutefois, les décisions concernant le pâturage dans les aires protégées doivent être prises au cas par cas. En Australie, des recherches approfondies ont montré que le pâturage du

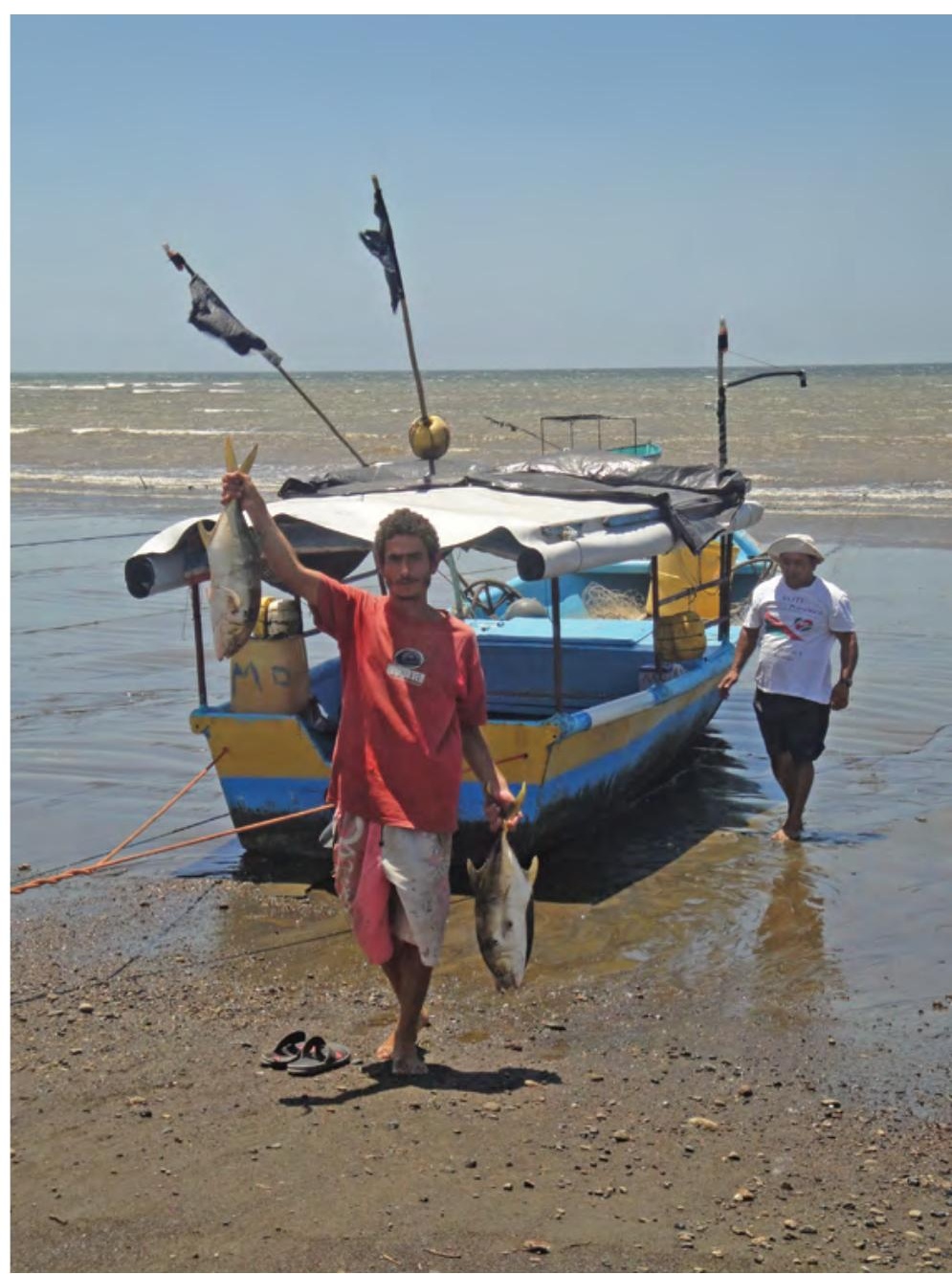

Aire marine de pêche responsable de Tárcoles, Costa Rica

Source : CoopeSolidar RL 


\section{Étude de cas 25.9 Lutte contre les menaces externes à l’̂̂le de Coron, aux Philippines}

Souvent, les luttes communautaires pour maintenir ou relancer l'utilisation durable ont été intégrées ou ont conduit à des luttes politiques plus générales pour les droits et le contrôle. C'est particulièrement le cas de nombreuses APAC. Les Tagbanwa des Philippines habitent la magnifique île calcaire de Coron, pour laquelle ils ont établi des règlements d'utilisation stricts (Ferrari et de Vera, 2003) (voir la photo de la page de titre, chapitre 8). Les ressources forestières doivent y être utilisées uniquement à des fins domestiques. Tous les lacs d'eau douce sont sacrés, sauf un. L'accès à ces lacs est strictement interdit à tous, sauf à des fins religieuses et culturelles. Le seul lac accessible pour le tourisme, hautement réglementé, est le lac Kayangan.

Jusqu'à récemment, les droits territoriaux des Tagbanwa n'étaient pas légalement reconnus, entrainant des empiétements de la part de pêcheurs migrants, d'opérateurs touristiques, de politiciens à la recherche de transactions foncières et d'agences gouvernementales. Cela a conduit à l'appauvrissement des écosystèmes marins et des ressources. Au milieu des années 1980, les insulaires se sont organisés en Fondation Tagbanwa de l'île de Coron (TFCl), et ont commencé un plaidoyer pour récupérer le contrôle de la gestion de leurs ressources naturelles. Ils ont d'abord demandé un Accord d'intendance communautaire des forêts (CFSA), qui a été accordé en 1990 pour les 7748 hectares de l'île de Coron et d'une île voisine, Delian, mais pas pour les zones marines. Les Tagbanwa ont poursuivi leur lutte et, en 1998, ont obtenu un Certificat de revendication de domaine ancestral pour 22284 hectares de terres et de zones marines. Enfin, en 2001, après avoir produit une carte de haute qualité et un Plan de gestion des terres ancestrales, ils ont réussi à obtenir un Certificat de titre de domaine ancestral (CADT), qui accorde des droits collectifs sur les terres. bétail cause des dommages importants à la végétation, aux sols et aux cours d'eau autochtones alpins et subalpins dans le parc national de Kosciuszko et dans le parc national Alpin (Williams, 1990 ; Wahren et al., 1994). Dans certaines aires protégées, les pressions exercées pour augmenter la production agricole ont conduit au surpâturage, mais plutôt qu'une interdiction, des mesures participatives ont été développées pour minimiser les dommages (étude de cas 25.7). Dans de nombreuses APAC (voir le chapitre 7), comme par exemple les forêts communautaires d'Asie du Sud, les communautés réglementent volontairement le pâturage par un arrêt temporaire ou saisonnier de toute activité de pâturage, permettant ainsi la régénération des paysages dégradés.

\section{Utilisation des ressources côtières et marines}

Les écosystèmes côtiers des aires marines protégées (AMP) accueillent souvent une importante utilisation des ressources, à des fins de subsistance et commerciales (Spalding et al., 2013). Les récoltes incluent des ressources comestibles comme les poissons, les mollusques, les mammiferes marins et les algues, des ressources de construction comme le bois de mangrove, les blocs de corail, le sable et la chaux, des ressources d'ornement comme les coquillages, les perles et les coraux, une utilisation scientifique incluant un large éventail d'espèces, un usage industriel, comme les palourdes géantes et les espèces produisant des produits pharmaceutiques, ainsi qu'une utilisation de mariculture comme les moules et les huîtres. De plus en plus, l'écotourisme et l'éducation sont des éléments importants de l'utilisation des environnements marins.

La préservation d'une pêche durable est, dans de nombreux cas, l'un des principaux objectifs de la désignation d'une AMP. L'AMP communautaire d'Eastport, sur l'Île de
Terre-Neuve, au Canada, était motivée par la dépendance historique de la communauté locale à l'égard de la pêche, après l'effondrement des stocks de poisson de fond et le déclin des prises de homard, comme moyen de protéger les stocks de ces derniers (Charles et Wilson, 2009). De même, une partie de la motivation des communautés dans l'établissement et la gestion du vaste réseau d'aires marines gérées localement (LMMA) dans le Pacifique Sud-Est était d'assurer un flux durable des avantages de la pêche dans ces régions (Govan, 2009).

Comme dans le cas de l'utilisation des ressources terrestres, les sociétés autochtones et traditionnelles ont mis en place des règlements en vertu du droit coutumier pour protéger les ressources marines de la surexploitation. Par exemple, en Corée, la plongée est traditionnellement une activité de femmes. Celles-ci se sont auto-réglementées, en convenant qu'elles n'utiliseraient pas d'équipement de plongée, même si ceux-ci étaient disponibles, de sorte que tout ce qu'elles peuvent prélever se limite à ce qu'elles peuvent rassembler en retenant leur souffle et en plongeant de manière traditionnelle. Elles collectent des poulpes, des ormeaux, des oursins, des limaces de mer, des concombres de mer et des algues, et vendent leurs produits depuis les années 1970 (Onishi, 2005 ; Pfeiffer, 2009).

Au Costa Rica, deux ans après la reconnaissance de l'aire marine de pêche responsable de Tárcoles, et les études présentées par les pêcheurs aux institutions de l'État, un permis d'utilisation durable d'une durée de trois mois pour la récolte de crevettes a été accordé aux communautés de pêcheurs locales (Madrigal Cordero et Solís Rivera, 2012). Dans de tels endroits, les systèmes autorégulés fonctionnent encore efficacement, mais souvent, des changements dans l'utilisation des terres et les régimes fonciers ont perturbé les pratiques en place depuis des millénaires. Dans le parc marin de Nosy Atafana, au nord-est de Madagascar, inclus dans la 


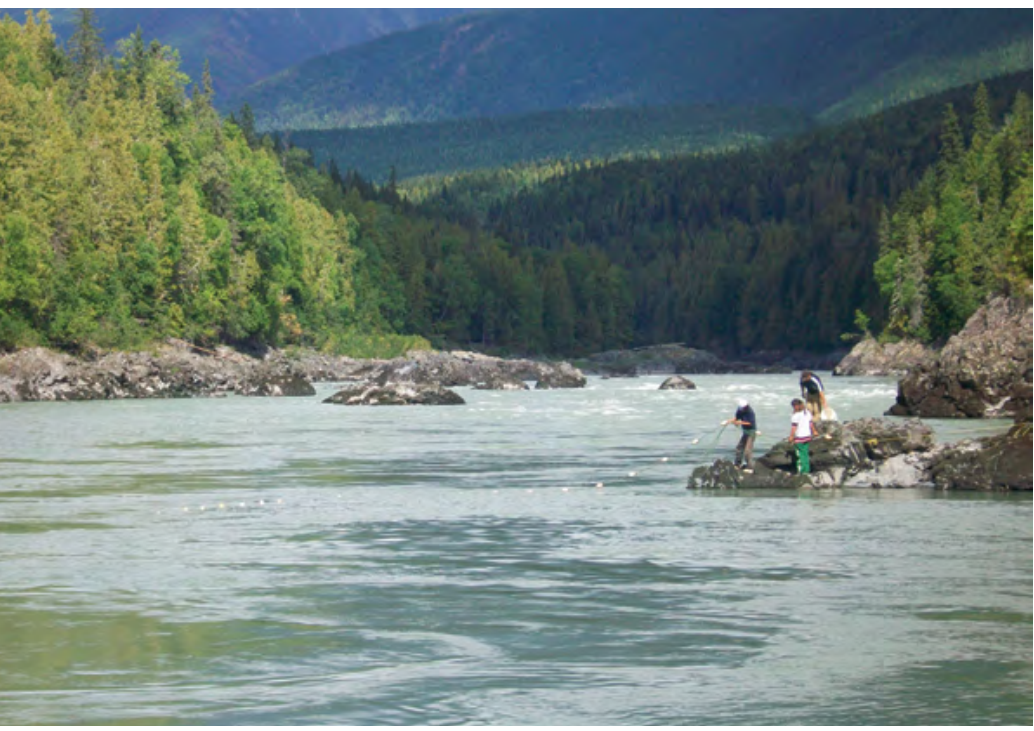

Rivière Skeena, Territoire Autochtone Gixtsan, Canada

Source : François Depey

réserve de biosphère de Mananara-Nord de l'Organisation des Nations unies pour l'éducation, la science et la culture (UNESCO), un accord entre les autorités de la réserve et les communautés locales précise les activités autorisées et interdites en ce qui concerne le poulpe, le concombre de mer et autres espèces de faune (IUCN, 2004).

Dans de nombreuses régions du monde, cependant, les AMP continuent d'être gérées de manière exclusive, entraînant des interdictions ou des restrictions même pour les utilisations traditionnelles non préjudiciables à la biodiversité, et autres façons de déposséder les communautés locales. L'un des résultats de cela est une hostilité croissante envers les AMP. Lorsqu'un processus de consultation et de négociation est en mesure de démontrer que les zones de non-capture peuvent aider à augmenter les populations de poissons en dehors de ces zones, il y a plus de chances que les gens acceptent toute une gamme de stratégies, y compris l'exclusion stricte de certaines zones ou pour une certaine période, comme par exemple, dans la réserve de biosphère de Seaflower, en Colombie (Friedlander et al., 2003). Dans certains cas, les communautés locales se sont organisées pour récupérer les droits perdus, comme les Tárcoles du Costa Rica mentionnées précédemment, puis pour élaborer leurs propres stratégies d'utilisation durable.

Le cas de Sainte-Lucie (étude de cas 25.8) illustre le chemin souvent difficile vers un accord et une utilisation durable des ressources dans une aire déclarée par le gouvernement, tandis que celui de l'Île de Coron (étude de cas 25.9) montre comment les peuples autochtones peuvent s'organiser contre les utilisations externes non durables pour conserver leurs APAC.

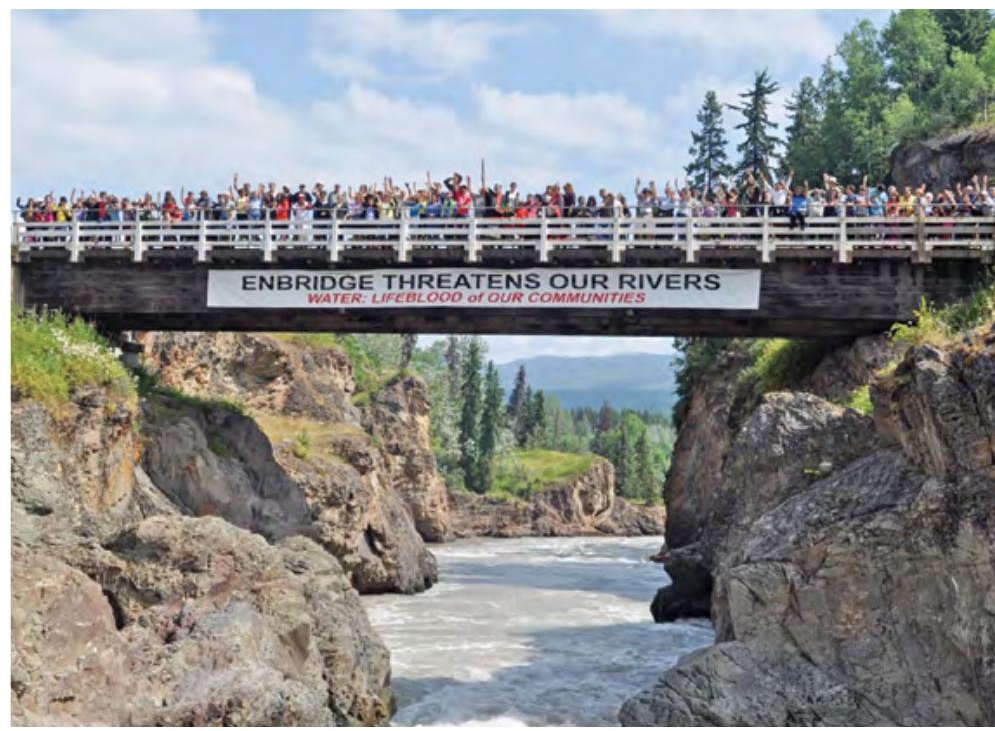

Résistance contre le projet de pipeline traversant les territoires autochtones des Skeenas

Source : Leah Macknak

\section{Changements mondiaux et utilisation des ressources}

Les impacts prévus des changements climatiques sur les aires protégées dans de nombreuses régions du monde obligeront à repenser leur rôle dans la conservation et l'utilisation durable de la biodiversité (Hunter et Heywood, 2011 ; voir aussi le chapitre 17). Les limites politiques des aires protégées sont fixes, mais le paysage biologique ne l'est pas. Il faudra repenser considérablement la conception de ces sites, ce qui aura des répercussions importantes sur la gestion des aires protégées (Schliep et al., 2008).

On s'attend à ce que le nombre de réfugiés environnementaux augmente d'environ 200 millions d'ici le milieu de ce siècle (Myers, 1997). Les impacts de cette situation sur la conservation et l'utilisation de la biodiversité pourraient être importants, dans la mesure où ces personnes migreront vers des territoires incapables de les soutenir ou de les nourrir sans perturbation à grande échelle. Cela entraînera également une augmentation des conflits entre les utilisateurs des ressources. De par leur nature même, les personnes déplacées seront fortement tributaires de leur environnement pour leur nourriture, leur bois de chauffage et autres besoins de subsistance, ce qui entraînera souvent une dégradation ou une perte des forêts et autres ressources (Hunter et Heywood, 2011).

La conservation et l'utilisation durable des ressources dans ces contextes nécessitera un changement de paradigme dans la façon dont nous abordons la gestion, la conception et la connectivité des aires protégées, et nécessitera des partenariats plus efficaces entre les administrations des aires protégées, les départements forestiers et agricoles travaillant 
dans les agro-écosystèmes traditionnels et les mouvements autochtones et sociaux (Perfecto et al., 2009 ; Padulosi et al., 2011).

\section{Développement et aires protégées}

Les projets de grande envergure inclus dans les stratégies nationales ou infranationales de croissance économique et de développement peuvent souvent se situer à l'intérieur ou à proximité d'une aire protégée. Ils comprennent l'exploitation minière et autres formes d'extraction, les projets d'hydroélectricité et d'irrigation, les routes et autoroutes, les ports, les installations sportives et touristiques, les lignes de communication et de transport d'électricité, l'expansion urbaine, etc. Bon nombre de ces projets constituent de graves menaces pour les écosystèmes et les espèces, ainsi que pour les populations humaines, dans les aires protégées.

Il existe peu d'évaluations nationales ou régionales du niveau et des types de menaces que le développement fait peser sur les aires protégées. L'évaluation nationale des aires protégées de l'Inde (Kothari et al., 1989) a révélé que $62 \%$ des 293 aires protégées étudiées contenaient au moins un des éléments suivants : routes, voies ferrées, mines, barrages, canaux, industries ou lignes de transmission. Une étude récente de l'ONG indienne Kalpavriksh a révélé qu'entre 1998 et 2009, près de 300 projets nécessitant la reconversion de terres à l'intérieur d'aires protégées ont été soumis à l'approbation du Conseil national de la faune du gouvernement central. Bien que beaucoup soient encore dans l'attente d'une décision, parmi ceux ayant été traités, la plupart ont été acceptés et peu ont été rejetés (Menon et al., 2010). Fait intéressant, presque toutes les propositions minières ont été approuvées, ce qui est difficile à comprendre, étant donné que l'exploitation minière est extrêmement dommageable.

Lune des questions les plus discutées en ce qui concerne le développement d'infrastructures affectant les aires protégées en Europe centrale est la construction d'autoroutes et de voies rapides. Les autoroutes et voies rapides traversent très souvent des habitats ou des corridors de migration importants d'espèces protégées telles que l'ours brun (Ursus arctos), le lynx (Lynx lynx), le chat sauvage ou le loup (Canis lupus). Findo et al. (2007) ont constaté que la majorité des collisions de véhicules avec des ours se sont produites pendant la période où les ours ont besoin de niveaux élevés d'aliments nutritifs, de la mi-juillet jusqu'à l'hibernation en novembre-décembre, lorsquils se déplacent sur de grandes zones traversées par des routes. Il en résulte que de nombreuses collisions entre les ours et les véhicules se produisent à l'intérieur et à l'extérieur des aires protégées.
Il s'agit d'un problème grave à l'échelle mondiale, bien que des progrès récents aient été réalisés dans la conception de passages surélevés et souterrains permettant un déplacement facilité de la faune d'un côté à l'autre (voir, par exemple, Locke, 2010).

En Amérique latine, pendant la majeure partie du siècle dernier, la déforestation était due à l'expansion de l'agriculture. Au cours des dernières années, cependant, la déforestation était, de plus en plus, le fait d'entités privées et de leurs activités. Avec l'augmentation de l'intensité des modes de consommation due à la mondialisation, la pression s'est accrue sur les aires protégées pour la production de biocarburants et de soja, d'énergie (géothermie et hydroélectricité), l'exploitation minière et le pétrole. De nombreux pays sont actuellement confrontés à d'énormes menaces de la part des gouvernements qui tentent de rétrograder, de réduire ou de déclasser des aires protégées (WWF, 2014). Par exemple, la production de soja en Argentine, au Paraguay et au Brésil, et plus récemment en Bolivie, a empiété sur plusieurs centaines de milliers d'hectares d'aires protégées. Les plantations d'huile de palme pour la production de biocarburants sont susceptibles d'être la principale cause de changements dans l'utilisation des terres en Asie tropicale, y compris dans les aires protégées par le gouvernement et sur de nombreuses terres autochtones et communautaires susceptibles de constituer des APAC (Campbell et al., 2008).

Les APAC et autres zones cruciales pour la conservation sont également menacées ailleurs. Au Chili, une bataille juridique, y compris au niveau de la Cour interaméricaine des droits de l'homme, a eu lieu entre le gouvernement et les groupes autochtones Mapuche-Pehuenche en raison de la construction de plusieurs barrages hydroélectriques sur la rivière BioBio (OLCA, 2014). Au Brésil, la construction du barrage de Belo Monte aura des conséquences dévastatrices sur une zone de plus de 1500 kilomètres carrés de forêt tropicale, et entrainera le déplacement forcé de 20000 à 40000 personnes (Washington Post, 2013). La nécessité d'une " énergie propre " (un terme inapproprié, étant donné que les grands réservoirs ont de graves impacts écologiques et climatiques) a été placée bien au-dessus de la nécessité de conservation et des droits des communautés. Le bassin versant de la Skeena, en Colombie-Britannique, au Canada, qui abrite plusieurs groupes autochtones et une faune importante, est menacé par des projets d'oléoducs et de gazoducs, de mines, de pêche commerciale, d'exploitation forestière et de lignes électriques (Skeena Watershed Conservation Coalition, 2014 ; SkeenaWild Conservation Trust, 2014).

Dans l'écozone du parc national du Grand Himalaya, en Inde (voir l'étude de cas 25.2), des changements écologiques importants sont causés ou menacés par des mégaprojets 
hydroélectriques, tels que le projet Parvati Hydel (Chhatre et Saberwal, 2006). Plus de 1000 hectares de terres forestières de choix dans l'aire protégée ont été reconverties pour le développement de l'hydroélectricité, et plusieurs autres grands et petits projets de ce type sur divers cours d'eau adjacents au parc restreignent encore les domaines vitaux de différentes espèces. Ceci est ironique, étant donné que les activités moins intrusives des villageois dans le PNGH adjacent ont été stoppées au nom de la conservation (voir l'étude de cas 25.2).

Les APAC, APP et autres initiatives de conservation sans statut officiel d'aire protégée ou reconnaissance officielle font face à des menaces encore plus grandes en raison des projets de développement et d'infrastructures (Borrini-Feyerabend et al., 2010 ; Kothari et al., 2012). Au moins, dans le cas des aires protégées officielles, la plupart des pays disposent de mécanismes juridiques ou politiques pouvant être utilisés pour réglementer ces développements, mais ce n'est pas le cas pour les sites et initiatives de conservation non reconnus.

En raison de la grande taille du réseau d'aires protégées d'Afrique de l'Est et d'Afrique australe, et de l'ampleur des besoins et des aspirations de développement économique de la région, la plupart des organismes de gestion des aires protégées disposent de cadres pour mettre en œuvre des plans de gestion généraux qui rationalisent le développement d'infrastructures telles que les routes, l'eau, les installations destinées au personnel, les installations et services touristiques, ainsi que la planification de la conservation et de la gestion des paysages. Ces cadres, souvent inscrits dans la législation nationale sur la faune sauvage ou les aires protégées, ont également tendance à définir des exigences en matière d'études d'impact environnemental et social (EIES), à réaliser lors de la planification du développement d'infrastructures dans les aires protégées. Les exigences et procédures d'EIES sont, à leur tour, un élément central des lois sur la gestion de l'environnement, qui, par exemple, ont été adoptées au Kenya, en Ouganda et en Tanzanie au cours des années 1990, et ont été progressivement mises en œuvre, à des degrés divers, dans toute la région (voir le chapitre 24).

La nature des processus et des résultats d'EIES, et la nature juridique de la législation environnementale en termes de responsabilisation des décideurs gouvernementaux en ce qui concerne les décisions et les impacts réglementaires sur l'environnement, jouent un rôle majeur dans les débats sur le développement d'infrastructures dans les principales aires protégées régionales. Le plus notable, ces dernières années, a été la proposition du gouvernement Tanzanien de construire une autoroute traversant la partie nord du parc national du Serengeti, afin de relier les différentes zones urbaines de cette partie du pays. Les biologistes craignent qu'une telle route ne conduise à une augmentation substantielle de la mortalité lors la migration annuelle nord-sud des gnous

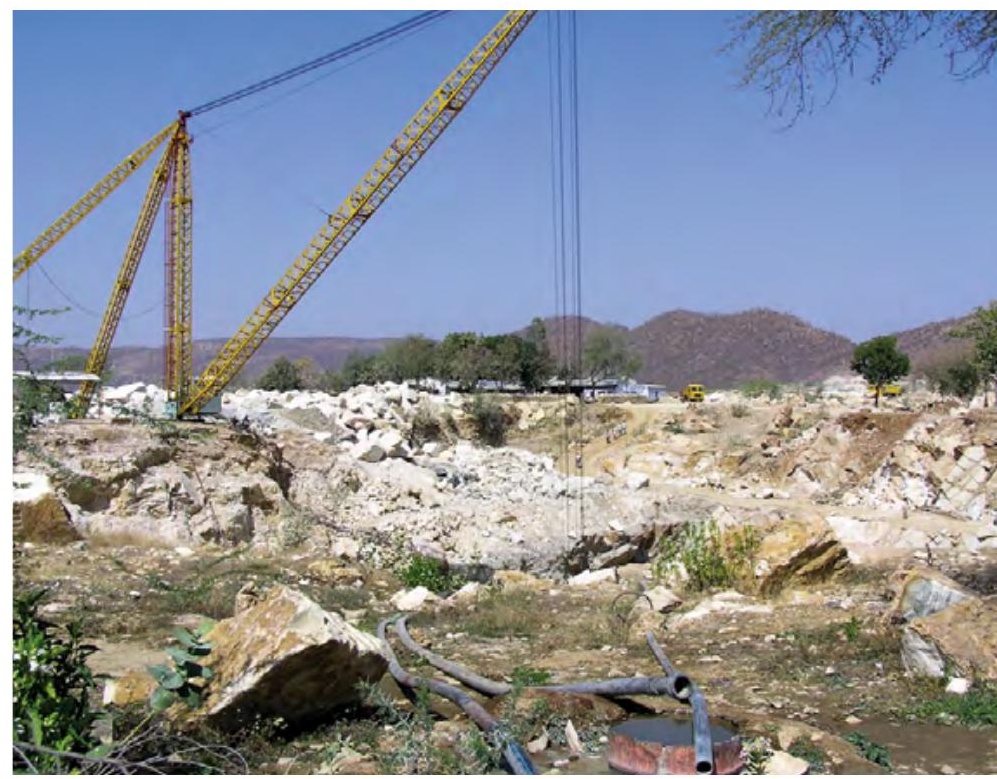

Exploitation minière à l'intérieur de la réserve de tigres de Sariska, Inde

Source : Ashish Kothar

entre les plaines du Serengeti et la réserve nationale Masai Mara, au nord du Kenya (Dobson et al., 2010 ; Norwegian University of Science and Technology, 2013). Un certain nombre de projets de compromis pour une route reliant les grandes populations humaines du lac Victoria aux centres urbains de l'Est du pays ont été proposés, et d'autres études d'EIES ont été commandées, le projet final de cette route étant encore très flou en 2014. Un débat similaire, bien que plus limité dans l'espace, a eu lieu au Kenya en 2010, autour d'un nouveau périphérique à Nairobi, qui aurait empiété sur les limites du parc national de Nairobi, à proximité de la capitale kényane. Cette route a récemment été contestée avec succès devant les tribunaux, et sa conception et construction finales sont maintenant incertaines (Koross, 2013).

Dans le sud de la Tanzanie, une importante nouvelle mine d'uranium a été développée sur des terres récemment déclassées de la réserve de faune du Selous (Tairo, 2014). La réserve du Selous est un site du Patrimoine mondial, dans lequel l'exploitation minière n'est pas autorisée, de sorte que le reclassement a d'abord dû être approuvé par le Comité du patrimoine mondial de l'UNESCO. Cependant, de tels processus soulèvent le spectre du déclassement d'autres aires protégées, même celles dont le niveau de reconnaissance internationale est élevé, lorsque des intérêts concurrents, commerciaux ou en matière d'infrastructures, entrent en jeu (voir l'étude de cas 25.2).

Dans un contexte mondial, il existe peu d'équilibre entre développement et conservation. Plus généralement, le contexte mondial de la croissance économique, l'augmentation des niveaux de consommation, la mondialisation économique et financière, les changements 
climatiques et autres facteurs similaires ont un impact sérieux sur divers écosystèmes. L'Évaluation des écosystèmes pour le millénaire, se concentrant sur les changements dans les écosystèmes ayant eu lieu à l'échelle mondiale au cours des 50 dernières années, prédit que les conséquences néfastes de la dégradation affectant la planète pourraient empirer au cours des 50 prochaines années (MEA, 2005). De nombreuses éditions du Global Biodiversity Outlook (Aperçu mondial de la biodiversité) (CBD, 2010) lancent des avertissements similaires.

La viabilité à long terme des aires protégées et des efforts de conservation dépendra de la mise en place de mécanismes institutionnels et d'interventions efficaces pour mieux s'attaquer aux causes réelles de la perte de biodiversité. Les autorités chargées des aires protégées sont généralement seulement responsables de la gestion à l'intérieur des réserves, même si la plupart des menaces émanent de l'extérieur des limites des aires protégées. Les gestionnaires des aires protégées devront donc travailler avec d'autres organismes et le secteur privé pour veiller à ce que les considérations relatives à l'intégrité et à la conservation des parcs soient intégrées à la planification locale et régionale. Dans certains cas, une seule agence pourrait être responsable à la fois de la gestion des aires protégées et de la planification du développement sectoriel, comme c'est le cas à Madagascar, où l'Agence nationale de gestion des aires protégées (ANGAP) est à la fois responsable du tourisme et des aires protégées. Ceci est toutefois plus une exception, qu'une règle. Les gouvernements locaux peuvent être des partenaires précieux pour s'assurer que la planification $\mathrm{du}$ développement complète les objectifs des aires protégées. Assurer une telle coopération exige non seulement de bonnes relations personnelles, mais aussi un appui et une coordination solides au niveau des États/provinces et entre les ministères au niveau national. Les gouvernements locaux sont plus susceptibles de soutenir la conservation lorsqu'ils reconnaissent les avantages que les aires protégées apportent pour stimuler la croissance économique locale (par exemple, le tourisme dans de nombreux pays), ou pour maintenir des fonctions écosystémiques cruciales comme l'approvisionnement en eau (par exemple, le parc national de Chingaza, en Colombie, source d'eau pour la capitale, Bogota ; voir Natural National Parks of Colombia, 2008).

Lune des rares tentatives pour y faire face systématiquement et au niveau mondial est le plaidoyer de l'UICN en faveur de la sauvegarde de certaines catégories d'aires protégées. Par exemple, lors du deuxième Congrès mondial de la nature de I'UICN (à Amman, Jordanie, en 2000), les membres ont adopté la Recommandation 2.82 (protection et conservation de la diversité biologique des aires protégées contre les impacts négatifs de l'exploitation et de l'exploration minière), qui : 1) appelle les États membres de l'UICN à interdire l'exploration et l'extraction minières dans les aires protégées des catégories I à IV, 2) recommande des contrôles stricts de ces activités dans les aires protégées des catégories $\mathrm{V}$ et VI, 3) demande instamment des normes strictes régissant les modifications des limites des aires protégées afin de permettre des activités minières, et 4) recommande des évaluations d'impact environnemental, afin de s'assurer que les activités minières en dehors des aires protégées n'aient pas d'impact négatif sur celles-ci.

Bien que cela n'ait pas été largement accepté, l'UICN et I'UNESCO ont réussi à convaincre le Conseil international des mines et des métaux (ICMM) d'accepter un moratoire volontaire sur l'exploitation minière dans les sites du Patrimoine mondial en 2003 (ICMM, 2003). Mais cela laisse de côté de nombreuses aires protégées tout aussi importantes pour la conservation. Le moratoire n'est que volontaire et susceptible d'être violé dans plusieurs pays où la gouvernance environnementale est faible. L'ICMM n'inclue pas toutes les sociétés minières, et, peut-être le point plus important, les aires protégées non officiellement reconnues (comme la plupart des APAC et des APP, comme indiqué ci-dessus et au chapitre 7) ne bénéficient d'aucune protection. Les critiques de l'industrie minière affirment que l'accord n'est qu'un " éco-blanchissement ", l'industrie ne devant assumer que peu ou pas de coûts mais gagnant en crédibilité. En outre, certains gouvernements sont souvent disposés à exciser des terres des limites des aires protégées pour permettre de telles activités (comme l'exemple du Selous mentionné précédemment). De nouveaux développements dans l'exploration pétrolière, y compris la fracturation hydraulique, menacent d'endommager encore plus les écosystèmes naturels dans de nombreuses parties $\mathrm{du}$ monde.

\section{Conclusion}

Quelques observations finales s'imposent pour les deux aspects abordés dans ce chapitre : l'utilisation des ressources et les projets de développement.

L'utilisation des ressources peut contribuer de plusieurs façons à la réalisation des objectifs de conservation, en termes écologiques (par exemple, lorsque les valeurs de la biodiversité sont maintenues par l'utilisation), économiques (par exemple, lorsque l'utilisation durable génère des revenus pour la gestion du parc) et sociaux (par exemple, lorsque l'utilisation durable locale renforce ou maintient le soutien local et l'assentiment pour la conservation). Les politiques et pratiques de conservation doivent être suffisamment souples pour tenir compte de l'utilisation des ressources par les communautés locales, en particulier lorsque celles-ci sont cruciales pour la survie et les moyens de subsistance, et 
sont ou peuvent être rendues compatibles avec les objectifs de conservation (en supposant que ces objectifs aient été définis de manière démocratique, en utilisant les meilleures connaissances disponibles et les principes et pratiques de «bonne gouvernance» décrits au chapitre 7). Cela a tendance à se produire souvent dans le cours naturel des événements, dans le cas des APAC, et dans une certaine mesure dans les aires protégées gérées conjointement, mais peut nécessiter une attention particulière dans de nombreuses aires protégées gérées par les gouvernements.

Lorsque cette utilisation des ressources ne peut en aucun cas être compatible avec les objectifs de conservation, des mesures ont été prises, dans de nombreux cas, pour imposer des restrictions et fournir ou faciliter simultanément des solutions alternatives (par exemple, dans le parc national de Kibale ; voir l'étude de cas 25.4). Cependant, ces solutions alternatives peuvent ne pas toujours compenser adéquatement les pertes (comme dans le cas du parc national du Grand Himalaya ; étude de cas 25.2), ou peuvent ne pas être culturellement appropriées et économiquement réalisables. Ces lacunes constituent une leçon clé des initiatives de PCDI dans diverses parties du monde (comme nous l'avons vu précédemment) et doivent être prises spécifiquement en compte dans la planification de la gestion des aires protégées.

Il est important de se rendre compte que chaque situation est unique. Ce qui fonctionne pour rendre l'utilisation durable des ressources dans un site peut ne pas fonctionner dans un autre. Certains points communs entre les sites et les situations peuvent être identifiés, et des enseignements peuvent être tirés entre les sites, mais pour chaque site et situation, une nouvelle évaluation, une étude et un suivi basés sur les connaissances locales et externes seront nécessaires. Les conflits inter et intra-communautaires, en particulier liés au régime foncier et à la rétention de terres, à l'accès aux ressources et à la répartition des avantages de ces utilisations, doivent être résolus pour que l'ensemble de la communauté participe effectivement à la conservation.

La sécurité foncière, les droits territoriaux, les droits sur les ressources, le droit de participer à la prise de décision et les responsabilités concomitantes à l'égard de la conservation et des autres peuples sont de plus en plus considérés comme essentiels pour la participation des populations locales à tous les types de gouvernance des aires protégées, ainsi que pour clarifier les rôles et responsabilités des organismes gouvernementaux dans le cas des aires protégées gérées par les gouvernements (voir le chapitre 7).

La gestion efficace des ressources doit reposer sur une bonne information, intégrée dans la science, les connaissances et les pratiques culturelles autochtones et traditionnelles, ou dérivée de méthodes scientifiques modernes. Les connaissances autochtones / traditionnelles et locales peuvent être d'une importance capitale pour cartographier les zones d'utilisation des habitats et des ressources, et établir des zones d'utilisation des ressources viables et socialement acceptables.

Il est important d'intégrer des stratégies pour faire faceà divers types de manque de privilèges sociaux et économiques, $y$ compris les inégalités entre hommes et femmes, les préjugés et inégalités ethniques, la capture des avantages par les élites et autres facteurs susceptibles de fausser la répartition équitable des pouvoirs et des capacités de prise de décision et des avantages en matière de conservation.

Des leçons peuvent être tirées des différents types de gouvernance. Par exemple, de nombreuses APAC ont élaboré des modes d'adaptation et des processus institutionnels pour déterminer les niveaux et types d'utilisation des ressources ne mettant pas en danger les écosystèmes et les espèces concernés, dont le gouvernement et autres pourraient tirer des enseignements. À l'inverse, de nombreuses aires protégées gérées par les gouvernements ont développé des systèmes robustes de planification de la gestion, dont les APAC pourraient tirer des enseignements. Aux niveaux national et infranational, des plateformes de partage et d'apprentissage doivent être créées.

Les facteurs mondiaux actuels et potentiels, tels que les changements climatiques, sont susceptibles de modifier la situation de l'utilisation des ressources à l'intérieur et autour des aires protégées. Une résilience et une flexibilité considérables, la connectivité entre les grands paysages terrestres et marins, et une collaboration entre les différents ayant-droits et parties prenantes seront nécessaires pour s'adapter à ces changements.

Les projets et processus de développement et d'infrastructures ayant un impact sur les aires protégées et autres sites de conservation doivent passer par des processus de sélection et de prise de décision démocratiques et fondés sur les connaissances, dans lesquels les autorités chargées des aires protégées et les populations locales devront avoir une voix centrale. Idéalement, les politiques nationales devront désigner les sites essentiels à des fins de conservation écologique et de la biodiversité comme des zones interdites aux activités à grande échelle ayant des impacts préjudiciables. Cela devrait également s'appliquer au « bassin versant » ou à la « zone d'impact » situés en dehors de l'aire protégée, où de telles activités pourraient avoir un impact sur l'aire protégée.

Si de telles mesures, ou des efforts plus globaux tels que le plaidoyer de l'UICN pour que certaines catégories d'aires protégées soient interdites à l'exploitation minière, sont des mesures visant à limiter les impacts négatifs 
du développement sur les aires protégées, l'accent est également de plus en plus mis sur la nécessité de réorienter le cadre de développement lui-même. Sans cela, les dommages écologiques généralisés, causés par l'industrie extractive, les infrastructures et autres processus de "développement " inhérents à un modèle qui place la croissance économique au-dessus de tout, continueront de saper à la fois la biodiversité et les communautés, en particulier celles qui dépendent le plus de l'environnement naturel. L'un des volets de cette réorientation est la voie du "développement durable ", dans laquelle les impacts environnementaux sont intégrés de manière plus centralisée dans la planification du développement, et l'économie s'oriente vers des processus, des technologies, une comptabilité et autres mesures plus écologiques. Tel est le sens de la déclaration issue de la Conférence Rio+20 - Conférence sur le développement durable (CDD, 2012), et des négociations en cours pour un programme pour l'après-2015 en remplacement des Objectifs du millénaire pour le développement (Sustainable Development Knowledge Platform, 2014). Un autre volet estime que cela sera insuffisant, car le développement reste tributaire de la croissance économique, impossible à soutenir dans un monde où prévalent des limites écologiques. Il appelle donc à un changement fondamental de direction vers des stratégies et des indicateurs de bien-être humains non basés sur la croissance, en phase avec les limites de la nature, et plus adaptés à la réalisation directe de la sécurité des besoins fondamentaux pour tous (eau, nourriture, abri, assainissement, vêtements, apprentissage, santé, relations sociales, etc.) (Rijnhout et al., 2014). Ce chapitre n'est pas l'endroit idéal pour aborder ces questions en détail, mais les gestionnaires d'aires protégées, les ayantdroits, les parties prenantes et tous ceux qui s'intéressent à la conservation devront s'engager d'une manière ou d'une autre dans ce contexte plus général de voies de développement et de bien-être.

\section{Références}

DD Lectures recommandées

Adams, W. M. (2004) Against Extinction: The story of conservation, Earthscan, Londres.

Alers, M., Bovarnick, A., Boyle, T., Mackinnon, K. et Sobrevila, C. (2007) Reducing Threats to Protected Areas: Lessons from the field, The World Bank and United Nations Development Programme, New York.

Amazon Conservation Association (2013) Amazon Conservation Association. <www.amazonconservation. org/ourwork/livelihoods.html>

Amend, S. et Amend, T. (1995) National Parks without People? The South American experience, IUCN and Parques Nacionales y Conservacion Ambiental, Quito.

10 Amend, T., Brown, J., Kothari, A., Phillips, A. et Stolton, S. (eds) (2008) Protected Landscapes and Agrobiodiversity Values, Values of Protected Landscapes and Seascape Volume 1, UICN et GTZ, Kasparek Verlag, Heidelberg.

Anderson, D. et Grove, R. H. (eds) (1987) Conservation in Africa: Peoples, policies and practice, Cambridge University Press, Cambridge.

Argumedo, A. (2008) 'The Potato Park, Peru: conserving agrobiodiversity in an indigenous biocultural heritage area', in T. Amend, J. Brown, Kothari, A. Phillips et S. Stolton (eds) Protected Landscapes and Agrobiodiversity Values, Values of Protected Landscapes and Seascape Volume 1, pp. 45-58, UICN et GTZ, Kasparek Verlag, Heidelberg.

Arshad, M. et Khalid, U. (2008) Ungulate population estimate of Torghar, Pakistan, manuscrit non publié.

Australian Committee for IUCN (1999) Great Barrier Reef World Heritage Area: Condition, management and threats, Australian Committee for IUCN, Sydney.

Bassi, M. et Tache, B. (2011) 'The community conserved landscape of the Borana Oromo, Ethiopia: opportunities and problems', Management of Environmental Quality: An International Journal 22(2): 174-86. 
Bassols Isamat, E., Perramon Ramos, B., Mallarach Carrera, J. M. et Falgarona Bosch, J. (2011) 'The conservation of the agrobiodiversity of La Garrotxa Volcanic Zone Natural Park', Management of Environmental Quality: An International Journal 22(2): 233-49. <www.emeraldinsight.com/journals. htm?issn $=1477-7835 \&$ volume $=22 \&$ issue $=2 \&$ PHPS $\mathrm{ESSID}=$ gnubmq2ks8b72aepclqldtg7v2>

Battharai, B., Ojha, H., Ram Banjade, M. et Luintel, H. (2003) Effect of NTFP Market Expansion on Sustainable Local Livelihoods, Forest Resource Action Team, Népal.

Baviskar, A. (2003) 'States, communities and conservation: the practice of ecodevelopment in the Great Himalayan National Park', in V. Saberwal et M. Rangarajan (eds) Battles over Nature: Science and the politics of conservation, Permanent Black, New Delhi.

Bélair, C., Ichikawa, K., Wong, B. Y. L. et Mulongoy, K. J. (eds) (2010) Sustainable use of biological diversity in socio-ecological production landscapes. Background to the 'Satoyama Initiative for the benefit of biodiversity and human well-being', Technical Series No. 52, Secretariat of the Convention on Biological Diversity, Montréal.

Berkes, F. (1989) Common Property Resources: Ecology and community-based sustainable development, Belhaven Press, Londres.

Bertzky, B., Corrigan, C., Kemsey, J., Kenney, S., Ravilious, C., Besançon, C. et Burgess, N. (2012) Protected Planet Report 2012: Tracking progress towards global targets for protected areas, UICN, Gland, et UNEP-WCMC, Cambridge.

Bezaury-Creel, J. et Gutiérrez Carbonell, D. (2009) Áreas naturales protegidas y desarrollo social en México, en Capital natural de México. Volume II: Estado de conservación y tendencias de cambio [Protected Areas and Social Development in Mexico: Natural capital of Mexico. Volume II: State of conservation and changing trends], Conabio, Mexique.

Bird, R. B., Tayor, N., Codding, B. F. and Bird, D. W. (2013) 'Niche construction and Dreaming logic: Aboriginal patch mosaic burning and varanid lizards (Varanus gouldii) in Australia', Proceedings of the Royal Society B: Biological Sciences 280(1772): 20132297.

Bolen, E. G. et Robinson, W. L. (2003) Wildlife Ecology and Management, 5th edn, Pearson Benjamin Cummings, San Francisco.
Borrini-Feyerabend, G., Kothari, A. et Oviedo, G. (2004) Indigenous and Local Communities and Protected Areas: Towards equity and enhanced conservation, IUCN WCPA Best Practice Series No. 11, UICN, Cambridge.

Borrini-Feyerabend, G. (2010) Strengthening what works: recognising and supporting the conservation achievements of indigenous peoples and local communities, Briefing Note No. 10, IUCN WCPA- CEESP TILCEPA, ICCA Consortium, CEESP TGER, GEF SGP, WAMIP, Cenesta, et GTZ, Gland.

Brandon, K., Redford, K. H. et Sanderson, S. E. (eds) (1998) Parks in Peril: People, politics and protected areas, The Nature Conservancy and Island Press, Washington, DC.

Brown, J. et Kothari, A. (2011) 'Traditional and agricultural landscapes and community conserved areas: an overview', Management of Environmental Quality: An International Journal 22(2): 139-53.

BurrenLIFE (2014) The BurrenLIFE Project, Carron, Irlande. <www.burrenlife.com/>

Cadman, M., Petersen, C., Driver, A., Sekhran, N., Maze, K. et Munzhedzi, S. (2010) Biodiversity for Development: South Africa's landscape approach to conserving biodiversity and promoting ecosystem resilience, South African National Biodiversity Institute, Pretoria. <www.undp.org/content/ undp/en/home/librarypage/ environment-energy/ ecosystems_and_biodiversity/ biodiversity_fordevelopment/>

Cairns, M. F. (2014) Shifting Cultivation and Environmental Change: Indigenous people, agriculture and forest conservation, Routledge, Oxford.

Campbell, A., Kapos, V., Lysenko, I., Scharlemann, J. P. W., Dickson B., Gibbs H. K., Hansen, M. et Miles, L. (2008) Carbon Emissions from Forest Loss in Protected Areas, UNEP World Conservation Monitoring Centre, Cambridge.

Campbell, L., Haalboom, B. et Trow, J. (2007) 'Sustainability of community-based conservation: sea turtle egg harvesting in Ostional (Costa Rica) ten years later', Environmental Conservation 34(2): 122-31.

Chamberlain, J. L., Cunningham, A. B. et Nasi, R. (2004) 'Diversity in forest management: non-timber forest products and bush meat', Renewable Resources Journal (Summer): 11-19. 
Charles, A. (2011) 'Human rights and fishery rights in small-scale fisheries management', in R. S. Pomeroy and N. L. Andrew (eds) Small-Scale Fisheries Management: Frameworks and approaches for the developing world, pp. 59-74, CAB International, Wallingford, Royaume-Uni.

Charles, A. et Wilson, L. (2009) 'Human dimensions of marine protected areas', ICES Journal of Marine Science 66: 6-15.

Chhatre, A. et Agrawal, A. (2009) 'Trade-offs and synergies between carbon storage and livelihood benefits from forest commons', Proceedings of the National Academy of Sciences 106(42): 17 667-70. <doi:10.1073/ pnas.0905308106>

Chhatre, A. et Saberwal, V. K. (2006) Democratizing Nature: Politics, conservation and development in India, Oxford University Press, New Delhi.

Chhetri, P., Mugisha, A. et White, S. (2003) 'Community resources use in Kibale and Mt Elgon National Parks, Uganda', Parks 13: 28-49.

Dd Child, B. (ed.) (2004) Parks in Transition: Biodiversity, rural development, and the bottom line, Earthscan, Londres.

Compton, J. et Le, Q. H. (1998) Borderline: An assessment of wildlife trade in Vietnam, WWF Indochina Programme, Hanoi.

Convention on Biological Diversity (CBD) (2004) Addis Ababa Principles and Guidelines, Convention on Global Biodiversity Secretariat, Montréal. <www.cbd.int/ sustainable/addis.shtml>

Convention on Global Biodiversity (CBD) (2010) Global Biodiversity Outlook, Convention on Global Biodiversity Secretariat, Montréal. <www.cbd.int/ $\mathrm{gbo} />$

Convention on International Trade in Endangered Species of Wild Fauna and Flora (CITES) (2000) Bushmeat as a trade and wildlife management issue, CoP11 Doc. 11.44, Eleventh Meeting of the Conference of the Parties, 10-20 April, Gigiri Kenya. <www.cites.org/ eng/cop/11/doc/44.pdf>
Convention on International Trade in Endangered Species of Wild Fauna and Flora (CITES) (2013) Status of African elephant populations and levels of illegal killing and the illegal trade in ivory: a report to the African Elephant Summit, December 2013, CITES Secretariat, IUCN/SSC African Elephant Specialist Group and TRAFFIC International, Genève.

Danielsen, F., Jensen, A. E., Alviola, P. A., Balete, D. S., Mendoza, M., Tagtag, A., Cistodio, C. et Enghoff, M. (2005) 'Does monitoring matter? A quantitative assessment of management decisions from locallybased monitoring of protected areas', Biodiversity and Conservation 14: 2633-52.

Das, P. (2007) 'The politics of participatory conservationthe case of Kailadevi Wildlife Sanctuary, Rajasthan', in G. Shahabuddin and M. Rangarajan (eds) Making Conservation Work, pp. 113-46, Permanent Black, New Delhi.

de Beer, F. (2013) 'Community-based natural resource management: living with Alice in Wonderland?', Community Development Journal 48(4 October): 555-70. <journals.ohiolink.edu/ejc/article.cgi?i ssn $=00103802 \&$ issue $=v 48 i 0004 \&$ article $=555$ cnrmlwaiw>

Desor, S. (2013) Citizens' report 2013 on community forest rights under Forest Rights Act, Kalpavriksh, Pune, and Vasundhara, Bhubaneshwar, with Oxfam India, Delhi, on behalf of Community Forest Rights Learning and Advocacy Process.

Dobson, A. P., Borner, M., Sinclair, A. R. E., Hudson, P. J., Anderson, T. M., Bigurube, G. et Davenport, T. B. B. (2010) 'Road will ruin Serengeti', Nature 467(7313): 272-3.

Dowie, M. (2009) Conservation Refugees: The hundred-year conflict between global conservation and native peoples, MIT Press, Cambridge, MA.

Drew, J. A. (2005) 'Use of traditional ecological knowledge in marine conservation', Conservation Biology 19(4): 1286-93.

Dudley, N. (ed.) (2008) Guidelines for Applying Protected Area Management Categories, UICN, Gland.

Evans, S. (1999) The Green Republic: A conservation history of Costa Rica, University of Texas Press, Austin. 
Failing, L., Gregory, R. et Harstone, M. (2007) 'Integrating science and local knowledge in environmental risk management: a decision-focused approach', Ecological Economics 64: 47-60.

FairWild (2009) FairWild Foundation, Cambridge et Zurich. <www.fairwild.org/>

Farvar, M. T. (2003) 'Myths, challenges and questions on mobile pastoralism in West Asia', Policy Matters 12: $31-41$.

Ferrari, M. F. et de Vera, D. (2003) 'A “participatory” or a "rights-based" approach? Which is best for protected areas and indigenous peoples in the Philippines?', Policy Matters 12: 166-70.

Find'o, S., Skuban, M. et Koreň, M. (2007) Brown Bear Corridors in Slovakia, Carpathian Wildlife Society, Slovaquie.

Food and Agriculture Organisation (FAO) (2010) Second Report on the State of the World's Plant Genetic Resources for Food and Agriculture, Food and Agriculture Organisation, Rome.

Food and Agriculture Organisation (FAO) (2012) The State of World Fisheries and Aquaculture, Food and Agriculture Organisation, Rome.

Fonseca, A. (2009) Decimosexto Informe Estado de la Nación en Desarrollo Sostenible. Informe Final. Estado de la Nación en la zona marino costera [Sixteenth State of the Nation in Sustainable Development. Final Report: State of the nation in coastal and marine areas], Proyecto Estado de La Nación Costa Rica, San José.

Friedlander, A., Sladek Nowlis, J., Sanchez, J. A., Appeldoorn, R., Usseglio, P., McCormick, C., Bejarano, S. et Mitchell-Chui, A. (2003) 'Designing effective marine protected areas in Seaflower Biosphere Reserve, Colombia, based on biological and sociological information', Conservation Biology 17(6): 1769-84.

Frisina, M. R. (2000) Suleiman markhor (Capra falconeri jerdoni) and Afghan urial (Ovis orientalis cycloceros) population status in the Torghar Hills, Balochistan Province, Pakistan, Unpublished report to the Society for Torghar Environmental Protection and the United States Fish and Wildlife Service.
Frisina, M. R. et Tareen, S. N. A. (2009) 'Exploitation prevents extinction: case study of endangered Himalayan sheep and goats', in B. Dickson, J. Hutton and B. Adams (eds) Recreational Hunting, Conservation and Rural Livelihoods: Science and practice, pp. 141-56, Wiley-Blackwell, Oxford.

Galland, P. (2013) Appraisal Report: Retezat National Park with extension to Piatra Craiului (Romania), Convention on the Conservation of European Wildlife and Natural Habitats, T-PVS/DE(2013)3, Council of Europe. <wcd.coe.int/ViewDoc. jsp?id=2042185\&Site=>

Galvin, M. et Haller, T. (2008) People, Protected Areas and Global Change: Participatory conservation in Latin America, Africa, Asia and Europe, NCCR North-South, University of Bern, Berne.

Gell, F. R. et Roberts, C. M. (2002) The Fishery Effects of Marine Reserves and Fishery Closures, WWF, Washington, DC.

Geoghegan, T. et Renard, Y. (2002) 'Beyond community involvement: lessons from the insular Caribbean', Parks (Special Issue on Local Communities and Protected Areas) 12(2): 16-26.

Gibson, C. C. (1999) Politicians and Poachers: The political economy of wildlife policy in Africa, Cambridge University Press, Cambridge.

Govan, H. (ed.) (2009) Status and Potential of Locally Managed Marine Areas in the South Pacific: Meeting nature conservation and sustainable livelihood targets through widespread implementation of LMMAs, Initiative for the Protection and Management of Coral Reefs in the Pacific, SPREP/WWF/ WorldFishReefbase/CRISP, Nouméa.

Govan, H., Jupiter, S. et Comley, J. (2012) 'Recognition and support of ICCAs in Fiji', in A. Kothari, with C. Corrigan, H. Jonas, A. Neumann et H. Shrumm (eds) Recognising and Supporting Territories and Areas Conserved by Indigenous Peoples and Local Communities: Global overview and national case studies, pp. 138-9, CBD Technical Series No. 64, Secretariat of the Convention on Biological Diversity, ICCA Consortium, Kalpavriksh and Natural Justice, Montréal.

Graves, A. (2004) Mapping Subsistence Agriculture in the National Park of American Samoa, National Park Service, US Department of the Interior, Washington, DC. <www.nps.gov/gis/mapbook/ tech/30.html> 
Gubbi, S., Linkie, M. et Leader-Williams, N. (2008) 'Evaluating the legacy of an integrated conservation and development project around a tiger reserve in India', Environmental Conservation 35: 331-9. <doi:10.1017/ S0376892908005225>

Gugić, G. (2009) Managing Sustainability in Conditions of Change and Unpredictability: The living landscape and floodplain ecosystem of the Central Sava River Basin, Lonjsko Polje Nature Park Public Service, Krapje, Croatie.

Hackel, J. D. (1999) 'Community conservation and the future of Africa's wildlife', Conservation Biology 13: 726-34.

Harper, S., Zeller, D., Hauzer, M., Pauly, D. et Sumaila, R. U. (2013) 'Women and fisheries: contribution to food security and local economies', Marine Policy 39: 56-63. <doi:10.1016/j. marpol.2012.10.018>

Hayes, T. M. (2006) 'Parks, people, and forest protection: an institutional assessment of the effectiveness of protected areas', World Development 34(12): 2064-75.

Heywood, V. H. (1999) 'Trends in agricultural biodiversity', in J. Janick (ed.) Perspectives on New Crops and New Uses, pp. 2-14, ASHS Press, Alexandria, VA.

Homewood, K. M. et Rodgers, W. L. (1991) Maasailand Ecology: Pastoralist development and wildlife conservation in Ngorongoro, Tanzania, Cambridge University Press, Cambridge.

Hunter, D. et Heywood, V. H. (2011) Crop Wild Relatives: A manual of in situ conservation, Earthscan, Londres.

Infield, M. (2003) 'National parks as cultural entities', Policy Matters 13: 64-70.

International Council on Mining and Metals (ICMM) (2003) ICMM Position Statement on Mining and Protected Areas, International Council on Mining and Metals, London. <www.icmm.com/publication s/497ICMMPositionStatementonMiningandProtec tedAreas.pdfs

International Union for Conservation of Nature (IUCN) (2000) IUCN Policy Statement on Sustainable Use of Wild Living Resources, Resolution 2.29 of the first World Conservation Congress, UICN, Gland.
International Union for Conservation of Nature (IUCN) (2004) 'Themesheet I6: Octopus and sea cucumber fisheries', in Managing Marine Protected Areas: A toolkit for the Western Indian Ocean, IUCN Eastern African Regional Programme, Nairobi. <wiomsa.org/ mpatoolkit/Themesheets/ I6_Octupus_and_sea_ cucumber_fisheries.pdf>

International Union for Conservation of Nature (IUCN), United Nations Environment Programme (UNEP) et World Wildlife Fund (WWF) (1980) World Conservation Strategy: Living resource conservation for sustainable development, IUCN et WWF, Gland, et UNEP, Nairobi.

Jan, A. (1992) Review and Analysis of Forest Polices of Pakistan, Government of Pakistan, Islamabad.

Kamboj, V. P. (2000) 'Herbal medicine', Current Science 78(1): 35-9.

Kathe, W. (2011) 'The new FairWild standard-a tool to ensure sustainable wild-collection of plants', Medicinal Plant Conservation 14: 14-17.

Kathe, W., Pätzold, B., Leaman, D., Timoshyna, A., Newton, D., Khou, E., Kinhal, G., Sapkota, I. B., Pasha, M. K. S., Ndam, N., Melisch, R., Bundalo, S., Honnef, S., Osborn, T., Buitrón, X. et Liu, X. (2010) Wild for a Cure: Ground-truthing a standard for sustainable management of wild plants in the field, TRAFFIC International, Cambridge.

Koross, K. (2013) 'Bypass stopped from encroaching on Nairobi National Park', The Star [Nairobi], 31 mai. $<$ www.the-star.co.ke/news/article-122527/bypassstopped-encroaching-nairobi-national-park>

Kothari, A. (2010) 'The birds are back', The Hindu Sunday Magazine, 7 March. <hindu.com/thehindu/ mag/2010/03/07/stories/2010030750400700.htm>

Kothari, A. et Pathak, N. (2005) 'Tragopans and tribals: a Naga transformation', Sanctuary Asia 25(5): 60-3.

Kothari, A., Corrigan, C., Jonas, H., Neumann, A. et Shrumm, H. (eds) (2012) Recognising and Supporting Territories and Areas Conserved by Indigenous Peoples and Local Communities: Global overview and national case studies, CBD Technical Series No. 64, Secretariat of the Convention on Biological Diversity, ICCA Consortium, Kalpavriksh and Natural Justice, Montréal. 
Kothari, A., Pande, P., Singh, S. et Variava, D. (1989) Management of National Parks and Sanctuaries in India: A status report, Indian Institute of Public Administration, New Delhi.

Kothari, A., Singh, N. et Suri, S. (1995) 'Conservation in India: a new direction', Economic and Political Weekly 30(43) (28 octobre): 2755-66.

Lasgorceix, A. et Kothari, A. (2009) 'Displacement and relocation of local communities from protected areas in India: a synthesis and analysis of case studies', Economic and Political Weekly XLIV(49) (5 décembre): 37-47.

Leader-Williams, N., Baldus, R. D. et Smith, R. J. (2009) 'The influence of corruption on the conduct of recreational hunting', in B. Dickson, J. Hutton et B. Adams (eds) Recreational Hunting, Conservation and Rural Livelihoods, pp. 296-316, Wiley-Blackwell, Oxford.

Leida, R., de Zoysa, U., Kothari, A. et Healy, H. (2014) 'Towards a global agenda of sustainability and equity: civil society engagement for the future we want', UNEP Perspectives 12. <www. unep.org/ civil-society/Portals/24105/documents/ perspectives/ ENVIRONMENT_PAPERS_DISCUSSION_12. pdfs

Locke, H. (2010) 'Yellowstone to Yukon Connectivity Conservation Initiative', in G. L. Worboys, W. Francis et M. Lockwood (eds) Connectivity Conservation Management: A global guide, Earthscan, Londres.

Lockwood, M., Worboys G. L. et Kothari, A. (2006) Managing Protected Areas: A global guide, Earthscan, Londres.

McNeely, J. A. (1998) Mobilising Broader Support for Asia's Biodiversity: How civil society can contribute to protected area management, Asian Development Bank, Manille.

McNeely, J. A., Miller, K. R., Reid, W. V., Mittermeier, R. A. et Werner, T. B. (1990) Conserving the World's Biodiversity, The World Bank, Washington, DC, et UICN, Gland.

McShane, T. O. et Wells, M. P. (2004) Getting Biodiversity Projects to Work. Towards more effective conservation and development, Columbia University Press, New York.
25. Gestion de l'utilisation des ressources et du développement

Madrigal Cordero, P. et Solís Rivera, V. (2012)

'Recognition and support of ICCAs in Costa Rica', in A. Kothari, with C. Corrigan, H. Jonas, A. Neumann et H. Shrumm (eds) Recognising and Supporting Territories and Areas Conserved by Indigenous Peoples and Local Communities: Global overview and national case studies, pp. 127-29, CBD Technical Series No. 64, Secretariat of the Convention on Biological Diversity, ICCA Consortium, Kalpavriksh and Natural Justice, Montréal.

Margolius, R. et Salafsky, N. (2001) Is Our Project Succeeding? A guide to threat reduction assessment for conservation, Biodiversity Support Programme, Washington, DC.

Martin, G., Camacho Benavides, C., del Campo Garcia, C., Fonseca, S. A., Chapela Mendoza, F. et Gonzalez Ortiz, M. A. (2011) 'Indigenous and communityconserved areas in Oaxaca,

Mexico', Management of Environmental Quality: An International Journal 22(2): 250-66.

Menon, M., Kohli, K. et Samdariya, V. (2010) 'Diversion of protected areas: role of the Wildlife Board', Economic and Political Weekly XLV(26-27): 18-21.

Millennium Ecosystem Assessment (MEA) (2005) Millennium Ecosystem Assessment, Island Press, Washington, DC. <www.maweb.org>

Myers, N. (1997) 'Environmental refugees', Population and Environment 19(2): 167-82.

Naqizadeh, N., Abbas, D. et Farvar, T. (2012) 'Recognition and support of ICCAs in Iran', in A. Kothari, with C. Corrigan, H. Jonas, A. Neumann et H. Shrumm (eds) Recognising and Supporting Territories and Areas Conserved by Indigenous Peoples and Local Communities: Global overview and national case studies, pp. 132-4, CBD Technical Series No. 64, Secretariat of the Convention on Biological Diversity, ICCA Consortium, Kalpavriksh et Natural Justice, Montréal.

Naidoo, R., Weaver, L. C., Stuart-Hill, G. et Tagg, J. (2011) 'Effect of biodiversity on economic benefits from communal lands in Namibia', Journal of Applied Ecology 48(2): 310-16. <doi:10.1111 /j.1365-2664.2010.01955.x> 
Nasi, R., Brown, D., Wilkie, D., Bennett, E., Tutin, C., van Tol, G. et Christophersen, T. (2008) Conservation and Use of Wildlife-Based Resources: The bushmeat crisis, CBD Technical Series 33, Secretariat of the Convention on Biological Diversity, Montréal, and Center for International Forestry Research, Bogor.

Nasi, R., Taber, A. et van Vliet, N. (2011) 'Empty forests, empty stomachs? Bushmeat and livelihoods in the Congo and Amazon Basins', International Forestry Review 13(3): 355-68.

National Park Service (NPS) (2014) National Park of American Samoa: Laws and Policies. <www.nps.gov/ npsa/parkmgmt/lawsandpolicies.htm>

Natural National Parks of Colombia (2008) Parque Nacional Natural: Chingaza. <www. parquesnacionales. gov.co/PNN/portel/libreria/ php/decide. php?patron=02.02021202\&f_patron $=02.020212>$

Daughton-Treves, L., Holland, M. B. et Brandon, K. (2005) 'The role of protected areas in conserving biodiversity and sustaining local livelihoods', Annual Review of Environment and Resources 30: 219-52. <doi:10.1146/annurev. energy.30.050504.164507>

Nelson, A. et Chomitz, K. M. (2011) 'Effectiveness of strict vs. multiple use protected areas in reducing tropical forest fires: a global analysis using matching methods', PLoS One 6(8): e22722. <doi:10.1371/ journal.pone.0022722>

Nelson, F. (ed.) (2010) Community Rights, Conservation and Contested Land: The politics of natural resource governance in Africa, Earthscan, Londres.

Nelson, F. (2012) 'Natural conservationists? Evaluating the impact of pastoralist land use practices on Tanzania's wildlife economy', Pastoralism 2(1): 1-19.

Nelson, F., Lindsey, P. et Balme, G. (2013) 'Trophy hunting and lion conservation: a question of governance?', Oryx 47(4): 501-9.

Nelson, F., Nshala, R. et Rodgers, W. A. (2007) 'The evolution and reform of Tanzanian wildlife management', Conservation and Society 5(2): 232.

Neumann, R. P. (1998) Imposing Wilderness: Struggles over livelihood and nature preservation in Africa. Volume 4, University of California Press, Berkeley.
Ngoitiko, M. et Nelson, F. (2013) 'What Africa can learn from Tanzania’s remarkable Masai lands rights victory', The Guardian, 8 octobre. <www. theguardian.com/ global-development/poverty- matters/2013/oct/08/ africa-tanzania-masai-land- rights-victory>

Niassa Carnivore Project (2013) Niassa Carnivore Project. $<$ niassalion.org/index.php >

Norwegian University of Science and Technology (2013) 'Serengeti road divides biologists: will a road across the northern tier of Serengeti National Park ruin it?', ScienceDaily, 23 mai. <www.sciencedaily. com/ releases/2013/05/130523082921.htm>

Oates, J. (1999) Myth and Reality in the Rain Forest: How conservation strategies are failing in West Africa, University of California Press, Berkeley.

Observatorio Latinoamericano de Conflictos Ambientales (OLCA) (2014) Center for Latin American Environmental Conflicts, Santiago. <olca.cl/oca/chile/ region $11 /$ represas 56 .htm>

Od Oglethorpe, J. A. E. (ed.) (1999) Tenure and Sustainable Use, UICN, Gland.

Onishi, N. (2005) 'South Korea's "sea women” trap prey and turn tables', The New York Times, 15 février. <www. nytimes.com/2005/02/15/ international/asia/15udo. html?_r=0>

Ostrom, E. (1990) Governing the Commons: The evolution of institutions for collective action, Cambridge University Press, Cambridge.

Padulosi, S., Hunter, D., Jarvis, A. et Heywood, V. (2011) 'Underutilized crops and climate change- current status and outlook', in S. Yadav, B. Redden, J. L. Hatfield et H. Lotze-Campen (eds) Crop Adaptation to Climate Change, pp. 507-21, Wiley- Blackwell, Ames, IA.

Pandey, S. (2008) 'Linking ecodevelopment and biodiversity conservation at the Great Himalayan National Park, India: lessons learnt', Biodiversity and Conservation 17: 1543-71.

Pandey, S. et Wells, M. (1997) 'Ecodevelopment planning at India's Great Himalayan National Park for biodiversity conservation and participatory rural development', Biodiversity and Conservation 6(9): 1277-92. 
Parr, S., Dunford, B., Moran, J., Williams, B. et Conchuir, R. (2010) 'BurrenLIFE-farming for conservation in the Burren', in C. Belair, K. Ichikawa, B. Y. L. Wong et K. J. Mulongoy (eds) Sustainable Use of Biological Diversity in Socio-Ecological Production Landscapes: Background to the Satoyama Initiative for the Benefit of Biodiversity and Human Well-Being, pp. 142-8, CBD Technical Series No. 52, Secretariat of the Convention on Biological Diversity, Montréal.

Parrotta, J. A. et Trosper, R. L. (eds) (2012) Traditional Forest-Related Knowledge, Springer, New York.

Pathak Broome, N. et Dash, T. (2012) 'Recognition and support of ICCAs in India', in A. Kothari, with C. Corrigan, H. Jonas, A. Neumann and H. Shrumm (eds) Recognising and Supporting Territories and Areas Conserved by Indigenous Peoples and Local Communities: Global overview and national case studies, pp. 130-2, CBD Technical Series No. 64, Secretariat of the Convention on Biological Diversity, ICCA Consortium, Kalpavriksh and Natural Justice, Montréal.

Perfecto, I., Vandermeer, J. et Wright, A. (2009) Nature’s Matrix: Linking agriculture, conservation and food sovereignty, Earthscan, Londres.

Periyar Tiger Reserve (2012) India Eco Development Project, Periyar Tiger Reserve, Thekkadi, Kerala, Inde. <www. periyartigerreserve.org/indian.php>

Peruvian Trust Fund for National Parks and Protected Areas (PROFONANPE) (2012) Peruvian Trust Fund for National Parks and Protected Areas, Lima. <www. profonanpe.org.pe/index.php/en/projects>

Pfeiffer, C. (2009) 'Mermaids of the deep', Sydney Morning Herald Traveller, 7 février. <www. smh.com.au/travel/ activity/surfing-and-diving/ mermaids-of-the-deep20090204-7xbg.html>

Porter-Bolland, L., Ellis, E. A., Guariguata, M. R., RuizMallen, I., Negrete-Yankelevich, S. et Reyes-Garcia, V. (2012) 'Community managed forests and forest protected areas: an assessment of their conservation effectiveness across the tropics', Forest Ecology and Management 268 (mars): 6-17. <doi:10.1016/j. foreco.2011.05.034>

Posey, D. (ed.) (1999) Cultural and Spiritual Values of Biodiversity, United Nations Environment Programme, Nairobi, and Intermediate Technology Publications, Londres.
Pretty, J. (2002). 'People, livelihoods and collective action in biodiversity management', in T. O'Riordan et $S$. Stoll-Kleemann (eds) Biodiversity, Sustainability, and Human Communities: Protecting beyond the protected, pp. 61-86, Cambridge University Press, Cambridge.

Programa Estado de la Nación (2006) Duodécimo informe Estado de la Nación en Desarrollo Humano Sostenible/Programa Estado de la Nación [Twelfth State of the Nation in Sustainable Development/ Human Development Program State of the Nation], San José, Costa Rica. <www.estadonacion.or.cr/files/ biblioteca virtual/006/info6cap4.pdf>

Radachowsky, J., Ramos, V. H., McNab, R., Baur, E. H. et Kazakov, N. (2012) 'Forest concessions in the Maya Biosphere Reserve, Guatemala: a decade later', Forest Ecology and Management 268: 18-28. <doi:10.1016/j. foreco.2011.08.043>

Redmond, I., Aldred, T., Jedamzik, K. et Westwood, M. (2006) Recipes for Survival: Controlling the bushmeat trade, World Society for the Protection of Animals, Londres.

Reid, H. (2001) 'Contractual national parks and the Makuleke community', Human Ecology 29(2): 135-55.

Rijnhout, L., de Zoysa, U., Kothari, A. et Healy, H. (2014) 'Towards a global agenda of sustainability and equity: civil society engagement for the future we want', UNEP Perspectives 12 (mars): 1-12. <www.unep.org/post2015/Publications/ TowardsAGlobalAgendaOfSustainabilityAndEqui/ tabid/133352/Default.aspx>

Roe, D., Nelson, F. et Sandbrook, C. (eds) (2009) Community Management of Natural Resources in Africa: Impacts, experiences and future directions, Natural Resource Issues No. 18, International Institute for Environment and Development, Londres.

Rybanič, R., Šeffer, J. et Čierna, M. (1999) 'Economic valuation of benefits from conservation and restoration of floodplain meadows', in J. Šeffer et V. Stanová (eds) Morava River Floodplain Meadows_Importance, restoration and management, pp. 147-60, DAPHNEInstitute of Applied Ecology, Bratislava.

Saberwal, V., Rangarajan, M. et Kothari, A. (2001) People, Parks and Wildlife: Tracts for the times 14, Orient Longman, New Delhi.

Salm, R. V., Clark, J. R. et Siirila, E. (2000) Marine and Coastal Protected Areas: A guide for planners and managers, UICN, Gland. 
Sathurusinghe, A., Seneneyake, S. J. G. N., Wijesundara, D. S. A. et Wijesekara, G. A. W. (2010) 'Species management plan for Cinnamomum capparu-coronde Blume', in B. Marambe et A. Wijesekara (eds) Conservation and Utilization of Crop Wild Relatives of Sri Lanka, pp. 35-6, Department of Agriculture and Ministry of Environment and Natural Resources, Sri Lanka.

Schippmann, U., Leaman, D. et Cunningham, A. B. (2006) 'Cultivation and wild collection of medicinal and aromatic plants under sustainability aspects', in R. J. Bogers, L. E. Craker et D. Lange (eds) Medicinal and Aromatic Plants: Agricultural, commercial, ecological, legal, pharmacological and social aspects, Springer, Dordrecht.

Schliep, R., Bertzky, M., Hirschnitz, M. et StollKleemann, S. (2008) 'Changing climate in protected areas? Risk perception of climate change by biosphere reserve managers', GAIA 17(1): 116-24.

Schulte-Herbrüggen, B., Cowlishaw, G., Homewood, K. et Rowcliffe, J. M. (2013) 'The importance of bushmeat in the livelihoods of West African cash- crop farmers living in a faunally-depleted landscape', PloS One 8(8): e72807.

Shahabuddin, G. (2010) 'India ecodevelopment project: a fragmented legacy', in G. Shahabuddin Conservation Crossroads: Science, society and the future of India's wildlife, pp. 166-86, Permanent Black, Delhi.

Shriar, A. (1999) 'Resource conservation and rural neglect: an example from Petén, Guatemala', Delaware Review of Latin American Studies 1(1). <www.udel.edu/LASP/ vol1Avrum.html>

Skeena Watershed Conservation Coalition (2014) Skeena Watershed Conservation Coalition, Old Hazelton, British Columbia. <skeenawatershed. com>

SkeenaWild Conservation Trust (2014) SkeenaWild Conservation Trust, Terrace, British Columbia. $<$ skeenawild.org>

Spalding, M. D., Meliane, I., Milam, A., Fitzgerald, C. et Hale, L. Z. (2013) 'Protecting marine spaces: global targets and changing approaches', Ocean Yearbook 27: 213-48.

Spence, M. (1999) Dispossessing the Wilderness: Indian removal and the making of the national parks, Oxford University Press, New York.
Spenceley, A. (ed.) (2008) Responsible Tourism: Critical issues for conservation and development, Routledge, New York.

Steenkamp, C. et Uhr, J. (2000) The Makuleke Land Claim: Power relations and community-based natural resource management, International Institute for Environment and Development, Londres.

Stoll-Kleemann, S. et Welp, M. (2008) 'Participatory and integrated management of biosphere reserves', GAIA 17: 161-68.

Sustainable Development Knowledge Platform (2014) Sustainable Development Knowledge Platform. <sustainabledevelopment.un.org>

Tairo, A. (2014) 'Tanzania gives go ahead to uranium mining in Selous Game Reserve', eTurboNews, 13 mai. $<$ www.eturbonews.com/45745/tanzania- gives-goahead-uranium-mining-selous-game- reserve>

Tandon, V. (2002) Getting women to choose: case study on sustainable livelihoods and poverty alleviation, Mountain Forum E-Consultation for the UNEP/ Bishkek Global Mountain Summit, Bishkek, Kyrgyzstan, 29 October - 1 November.

Tebtebba Foundation (2008) Indicators Relevant for Indigenous Peoples: A resource book, Tebtebba Foundation, Baguio City, Philippines. <www.tebtebba. org/index.php/content/123-indicators- relevant-forindigenous-peoples-a-resource- book $\% 20 \% 20>$

Technical and Policy Core Group (TPCG) et Kalpavriksh (2005) Securing India's future: final technical report of the National Biodiversity Strategy and Action Plan, Technical and Policy Core Group and Kalpavriksh, Pune. <www.kalpavriksh. org/index.php/conservationlivelihoods1/72- biodiversity-and-wildlife/nationalbiodiversity- strategy-action-plan/224-nbsap-finaltechnical- report.html>

Terborgh, J. (2004) Requiem for Nature, Island Press, Washington, DC.

United Nations (UN) (2009) State of the World's Indigenous Peoples, United Nations, New York.

United Nations Conference on Sustainable Development (UNCSD) (2012) The Future We Want, A/Conf.216/L.1, United Nations, New York. <www.uncsd2012.org/content/ documents/774futurewewant_english.pdf> 
United Nations University Institute of Advanced Studies Operating Unit Ishikawa/Kanazawa (UNU- IAS OUIK) (2011) Biological and Cultural Diversity in Coastal Communities: Exploring the potential of satoumi for implementing the ecosystem approach in the Japanese Archipelago, Technical Series No. 61, Secretariat of the Convention on Biological Diversity, Montréal.

United States Fish \& Wildlife Service (2014) RefugeSpecific Hunting and Sport Fishing Regulations, National Wildlife Refuge System. <www.fws.gov/ refuges/ hunting/huntfishregs.html>

Unnikrishnan, P. M. et Suneetha, M. S. (2012) Biodiversity, traditional knowledge and community health: strengthening linkages, UNU-IAS Policy Report, United Nations University Institute of Advanced Studies, Yokohama, Japon. <www. ias.unu.edu/resource_centre/ Biodiversity $\% 20$ Traditional $\% 20$ Knowledge $\% 20$ and $\% 20$ Community $\% 20$ Health_final.pdf>

van Oudenhoven, F., Mijatovic, D. et Eyzaguirre, P. (2011) 'Social-ecological indicators of resilience in agrarian and natural landscapes', Management of Environmental Quality: An International Journal 22(2): 154-73.

van Vliet, N., Nasi, R., Abernethy, K., Fargeot, C., Kumpel, N. F., Obiang, A. N. et Ringuet, S. (2012) 'The role of wildlife for food security in Central Africa: a threat to biodiversity?', in C. de Wasseige, P. de Marcken, N. Bayol, F. Hiol-Hiol, P. Mayaux, B. Desclee, R. Nasi, A. Billand, P. Defourny et R. Eba'a Atyi (2012) The Forest of the Congo Basin: State of the forest 2010, pp. 123-35, Publications Office of the European Union, Luxembourg. <doi:10.2788/47210>

Vasundhara et Kalpavriksh (2012) A National Report on Community Forest Rights under Forest Rights Act: Status and issues. An output of the Community Forest Rights Learning and Advocacy Process, Vasundhara, Bhubaneshwar and Kalpavriksh, Delhi et Pune.

Vaughan, C., McCoy, M., Fallas, J., Cháves, H., Barboza, G., Wong, G., Rau, J., Carranza, M. et Carbonell, M. (1996) Management and Development for Palo Verde National Park and Lomas Barbudal Biological Reserve, National University of Costa Rica, Heredia.

Vijayan, V. S. (1991) Keoladeo National Park Ecology Study: 1980-90. Final report, Bombay Natural History Society, Bombay.
Wahren, C.-H., Papst, W. A. et Williams, R. J. (1994) 'Long-term vegetation change in relation to cattle grazing in subalpine grassland and heathland on the Bogong High Plains: an analysis of vegetation records from 1945 to 1994', Australian Journal of Botany 42: 607-39.

Wani, M. et Kothari, A. (2007) 'Protected areas and human rights in India - the impact of the official conservation model on local communities', Policy Matters 15: 100-14.

Washington Post (2013) 'In Brazil, Amazonian Indians protest hydroelectric dam', Washington Post, 27 mai. <www.washingtonpost.com/world/ in-brazil-amazonian-indians-protest-hydroelectricdam/2013/06/10/f90539b6-cee5-11e2-8845d970ccb04497_gallery.html\#photo=1>

Wells, M., Brandon, K. et Hannah, L. (1992) People and Parks: Linking protected area management with local communities, The World Bank, WWF et US Agency for International Development, Washington, DC.

Wells, M., Guggenheim, S., Khan, A., Wardojo, W. et Jepson, P. (1999) Investing in Biodiversity: A review of Indonesia's integrated conservation and development projects, The World Bank, Washington, DC.

Dest, P., Igoe, J. et Brockington, D. (2006) 'Parks and peoples: the social impact of protected areas', Annual Review of Anthropology 35: 251-77.

Western, D. et Gichohi, H. (1993) 'Segregation effects and the impoverishment of savanna parks: the case for ecosystem viability analysis', African Journal of Ecology 31(4): 269-81.

Wieting, R. (2004) Sustainable Land Use in European Protected Areas, IUCN Regional Office for Europe, Bruxelles.

Wikipedia (2014) Niassa Reserve. <en.wikipedia.org/ wiki/ Niassa_Reserve>

Wildlife Institute of India (WII) (1999) An Ecological Study of the Conservation of Biodiversity and Biotic Pressures in the Great Himalayan National Park Conservation Area: An ecodevelopment approach. Volumes 1-6, Final Report, Forestry Research Education and Extension ProjectGreat Himalayan National Park, Wildlife Institute of India, Dehradun. 
Williams, R. J. (1990) 'Cattle grazing within subalpine heathland and grassland communities on the Bogong High Plains: disturbance, regeneration and the shrubgrass balance', Proceedings of the Ecological Society of Australia 16: 255-65.

Wittmer, H. U., Mark Elbroch, L. et Marshall, A. J. (2013) 'Good intentions gone wrong: did conservation management threaten endangered huemul deer Hippocamelus bisulcus in the future Patagonia National Park?', Oryx 47(3): 393-402. <doi:10.1017/ S0030605312000531>

World Resources Institute (WRI), International Union for Conservation of Nature (IUCN) et United Nations Environment Programme (UNEP) (1992) Global Biodiversity Strategy: Guidelines for action to save, study, and use Earth's biotic wealth sustainably and equitably, WRI, IUCN et UNEP, Gland.

World Wildlife Fund (WWF) (2014) PADDDtracker: Tracking protected area downgrading, downsizing, and degazettement. <www.PADDDtracker.org>

Zinn, C. et Vidal, J. (1999) 'How prawn cocktails and tourists are taking the life out of the Great Barrier Reef, The Guardian, 14 janvier. <www. theguardian. com/world/1999/jan/14/johnvidal> 
Ce texte est extrait du livre "Gouvernance et gestion des aires protégées " édité par Graeme L. Worboys, Michael Lockwood, Ashish Kothari, Sue Feary et Ian Pulsford, publié en 2020 par l'ANU Press, l'Australian National University, Canberra, Australie.

La reproduction de cette publication de l'ANU Press à des fins éducatives ou non commerciales est autorisée sans l'autorisation écrite préalable du détenteur des droits d'auteur, à condition que la source soit clairement indiquée. La reproduction de cette publication pour la revente ou à d'autres fins commerciales est interdite sans l'autorisation écrite préalable du détenteur des droits d'auteur. 